\title{
Hydrogeochemical evaluation and mechanisms controlling groundwater in different geologic environments, Western Sokoto Basin, Northwestern Nigeria
}

\author{
Saadu Umar Wali ${ }^{1}$. Noraliani Alias ${ }^{1} \cdot$ Sobri Bin Harun ${ }^{1}$
}

Received: 11 May 2020 / Accepted: 24 September 2020 / Published online: 10 October 2020

(c) Springer Nature Switzerland AG 2020

\begin{abstract}
The hydrogeochemistry of aquifers in the western Sokoto basin was assessed. The objective of this study is to identify the impact of geological variability on groundwater hydrochemistry and the mechanisms controlling the hydrochemistry of aquifers. Univariate statistics (several samples ANOVA), Pearson's $(r)$, and multivariate statistics-hierarchical cluster analysis (HCA) and Factor analysis (FA) were used to interpret the hydrochemistry of aquifers. Subsequently, geochemical modeling was applied to assess the saturation index (SI) of rock minerals. Forty groundwater samples were collected from Gwandu $(n=20)$ and IIlo $(n=20)$ formations. ANOVA results indicated that geological variability exerted a considerable impact on groundwater in Gwandu and Illo aquifers. It is characterized by a substantial amount of $\mathrm{Ca}^{2+}, \mathrm{Mg}^{2+}$, $\mathrm{SO}_{4}{ }^{2-}, \mathrm{HCO}_{3}{ }^{-}, \mathrm{Na}^{+}$, and $\mathrm{K}^{+}$. The hydrogeochemical facies indicated mixing conditions. FA and correlations analysis further revealed that groundwater received the noticeable influence of silicate weathering and dissolution of carbonates. There were significant differences in $\mathrm{SI}$ values for chrysotile, goethite, gypsum, $\mathrm{H}_{2}(\mathrm{~g}), \mathrm{H}_{2} \mathrm{O}(\mathrm{g}), \mathrm{H}_{2} \mathrm{~S}(\mathrm{~g})$, illite, and sepiolite minerals between the two geologic environments. Positive SI values for gibbsite were noticed in eleven sampling locations, indicative of potential recharge zones. Likewise, all the sampling locations have positive values for K-feldspar and are supersaturated with mica, suggesting both discharge and transition zones. The HCA classified aquifers into three clusters based on their hydrogeochemical characteristics. Geochemical modeling, univariate, and multivariate analyses are user-friendly tools for hydrochemical analysis.
\end{abstract}

Keywords Rock weathering $\cdot$ lon exchange process $\cdot$ Saturation index $\cdot$ Schoeller index $\cdot$ Anthropogenic input $\cdot$ Sodium adsorption ratio

\section{Introduction}

Increasing urbanization, industrialization, and irrigation farming in developing countries have imposed great pressure on groundwater resources, with its consequent depletion and/or permanent contamination of aquifers [9, $35,60,76,95]$. The hydrochemistry of aquifers is primarily influenced by geology, land use, quality of recharge water, soil-water interactions, recharge pathways, the residence time of recharged water in aquifers, and aquifer rock-water reactions. Therefore, local or regional groundwater chemistry is affected by local anthropogenic activities and inherent hydrogeochemical configurations [29, $31,80]$. The hydrochemistry of some groundwater aquifers is naturally excellent, but over time, their composition can be modified by anthropogenic activities and to a less significant extent by natural factors.

Saadu Umar Wali, saadu.umar@graduate.utm.my; Noraliani Alias, noraliani@utm.my; Sobri Bin Harun, sobriharun@utm.my|

${ }^{1}$ Department of Water and Environmental Engineering, School of Civil Engineering, Faculty of Engineering, Universiti Teknologi Malaysia, 81310 UTM Skudai, Johor, Malaysia. 
Evaluation of hydrogeochemical properties of groundwater plays a significant role in defining the suitability of groundwater for domestic, industrial, and irrigation use $[17,92,97,102,103]$. It provides a better understanding of the hydrochemical composition of groundwater aquifers [84]. Municipal and industrial wastes, in addition to the application of chemical fertilizers and pesticides, have emerged as foremost groundwater pollutants in heavily irrigated areas. Anthropogenic activities alter the natural source of contaminants and consequently initiate pollution load-receiving sub-surface aquifers $[96,98]$. Therefore, the assessment of the hydrochemical properties of aquifers is justifiable, as, in some remote areas, groundwater is the only source of water supply, especially in developing countries $[25,30,47,86]$. Groundwater suitability for drinking and irrigation uses can be determined by its physical and chemical properties. It can be revealed by hydrochemical analysis. The standard for the classification of groundwater for different uses is unique. Studies characterizing groundwater were carried out in different parts of the world $[3,34,42,50,74,79,90,101,114]$. Results have indicated anthropogenic inputs through variation in total dissolved solids (TDS), chloride $\left(\mathrm{Cl}^{-}\right)$, sulfate $\left(\mathrm{SO}_{4}{ }^{2-}\right)$, nitrate $\left(\mathrm{NO}_{3}{ }^{-}\right)$, and sodium $\left(\mathrm{Na}^{+}\right)$concentrations. Likewise, many studies have observed that rock weathering, aridity, and saltwater intrusion, are the major controls on hydrochemistry of aquifers $[4,32,71,91,106,115]$.

To highlight this problem, an investigation was carried out at the western Sokoto basin in Northwestern Nigeria. Groundwater studies in Sokoto basin have shown that the aquifers contained Holocene aged water (i.e., $100-10,000$ years BP). It is hard, and TDS varied from 130 to 2340 (mg/l). Nitrate and $\mathrm{Na}^{+}$vary widely, at some locations above WHO reference guidelines [12]. Groundwater is chiefly of two facies; $\mathrm{Ca}-\mathrm{Mg}-\mathrm{SO}_{4}-\mathrm{Cl}$ and $\mathrm{Ca}-\mathrm{Mg}-\mathrm{HCO}_{3}$ water type [6-8]. The hydrogeochemical facies are derived from the dissolution of carbonate ions (calcite and dolomite). More than $1 / 4$ of global population depended on carbonate aquifers for water supply. The geochemical assessment of these types of water-bearing formations is important for the protection and management of regional groundwater resources [49]. Groundwater in Gwandu Formation is soft with moderate TDS. However, groundwater properties of Illo formation are poorly known.

Characterization of groundwater over different geologic environments helps in solving diverse hydrochemical problems, especially if water quality is concerned. Thus, it is vital to understand aquifer hydrogeochemistry, for water quality management and effective development and sustainable utilization of freshwater. Studies relating hydrochemistry of aquifers with geological variability showed that changes in mineralogy and lithology significantly influenced changes in chemical composition and hydraulic properties of aquifers [54]. The spatial variation of groundwater properties and its evolution are regulated by the regional geology [10]. Variation in groundwater hydrochemistry is correlated to the local geology, resulting in distinct hydrochemical processes [64]. The objective of this study is to assess the impact of geological variability on the hydrochemical composition of groundwater in the study area.

\section{The geography of the study area}

\subsection{Location, size, and climate}

Sokoto basin, otherwise known as the lullemmeden basin (Fig. 1), is part of an extensive elongated sedimentary basin in west Africa. It covered most of Northwestern Nigeria and eastern parts of the Niger Republic. The basin is located between Latitude $10^{\circ}$ and $14^{\circ} \mathrm{N}$ and Longitude $3^{\circ}$ to $7^{\circ} \mathrm{E}$ [13]. The Sokoto basin is encircled southward and westward by the Republic of Niger. It is in the driest and hottest parts of West Africa since it is situated above $10^{\circ} \mathrm{N}$, within the Sahel Region of Africa that is frequently affected by droughts [83]. The study area has an AW Tropical climate. Temperature is generally high, and the average minimum temperature is $16^{\circ} \mathrm{C}$. The mean maximum temperature reaches a peak of $40^{\circ} \mathrm{C}$ in April. Rainfall is highly variable and varies between $500 \mathrm{~mm}$ in the northern parts by over $2500 \mathrm{~mm}$ in the south. There is an overall decline in relative humidity from south to north. The relative humidity is also highly variable and reaches the peak $(>90 \%)$ in August. It falls to desert-like conditions (10-30\%) during the dry season. The rate of evaporation is high and potential evaporation exceeds $2500 \mathrm{~mm}$ per annum [13, 38].

\subsection{Hydrogeology}

Geological analysis in the Sokoto basin began in the 1880 s. It focused initially on depicting the general geologic interpretations and reporting of fossil fuel regions. An allinclusive stratigraphy of the basin was initially depicted by Jones [56] and trailed by Parker et al. [85]. The geology of the Sokoto basin is detailed in the literature $[58,59,77,78]$. Similarly, the hydrogeology of the Sokoto basin is also well reported by previous studies. There are published data on hydrogeology and hydrogeochemistry of the Sokoto basin. Major aquifers are Gwandu, Illo, Kalambaina, Wurno, Taloka, Gundumi, Dukamaje, and Dange formations. The Basement Complex outcropped in the east and southwest $[11,13]$. A theorized hydrogeological setting of the Sokoto basin is presented in Fig. 2 . 


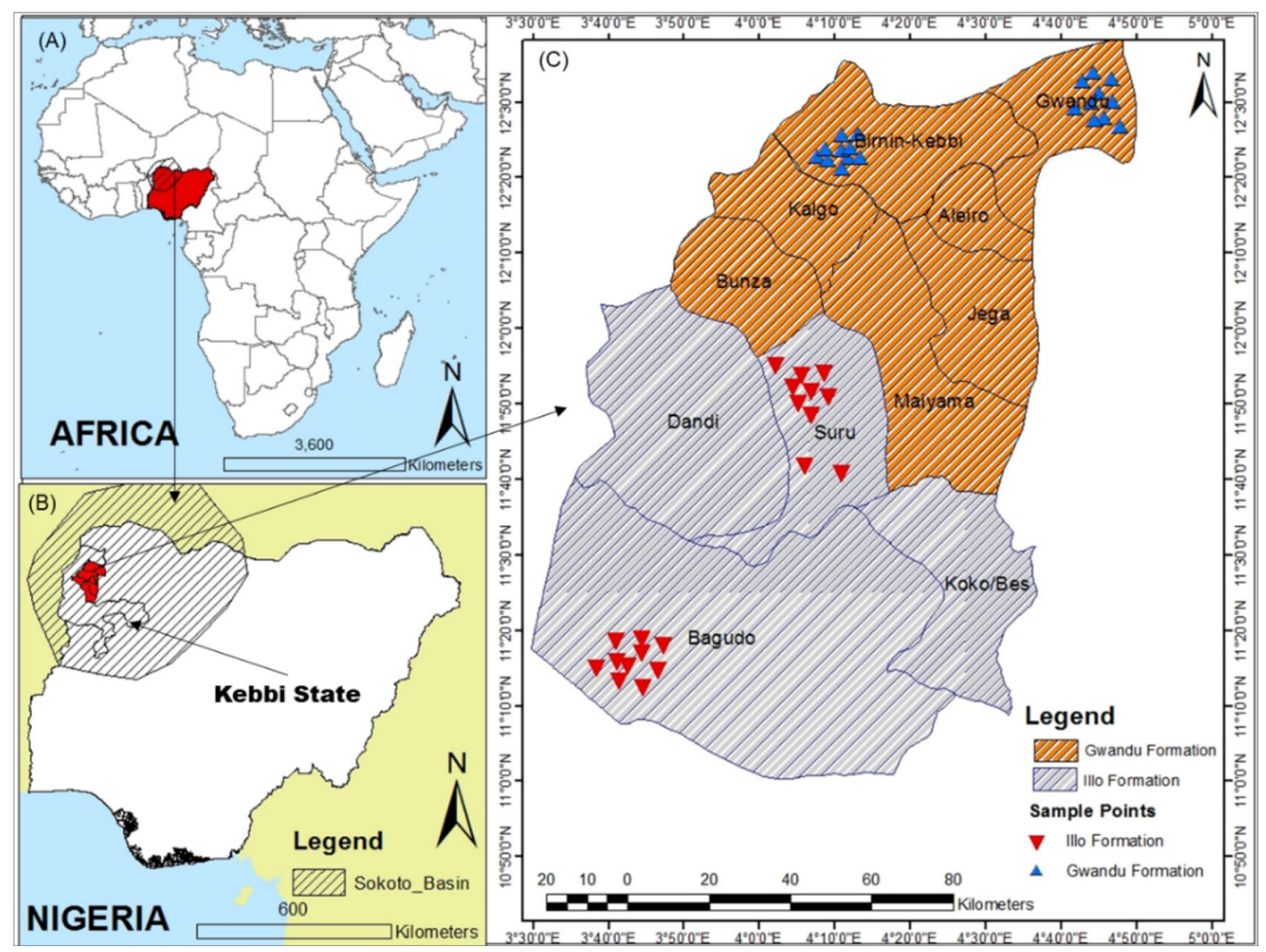

Fig. 1 Location of the study area

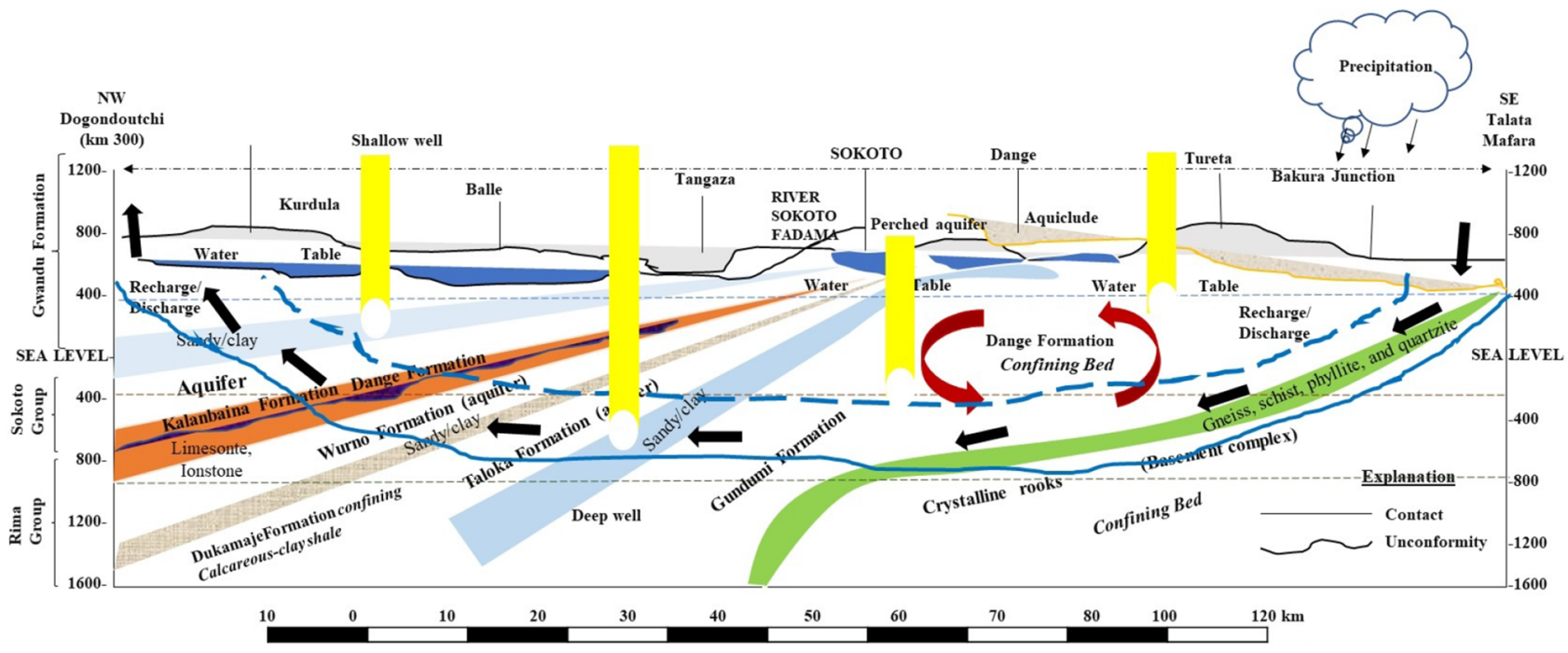

Fig. 2 Theorized hydrogeological cross section of the Sokoto basin, NW Nigeria, illustrating primary confining beds and groundwater aquifers in the study area 


\subsubsection{The Gwandu formation}

The Gwandu formation is the best-known aquifer in the Sokoto basin. It is continental in origin and consists of interbedded, moderately consolidated sands and clays. The clay beds are chiefly thick, massive, white, red grey black, and brown $[82,83]$. The sands vary from fine to coarse in texture. Gwandu aquifer is more essential, due to the basal sands, well saturated with water and confined by the underlying $16 \mathrm{~m}$ clay of Kalambaina formation and the overlying $10 \mathrm{~m}$ of clay of the Gwandu formation. The Gwandu formation contained two aquiferous zones: the uppermost unconfined sandy sections and the lower-most basal confined sandy zones. Recharge into the aquifer is by infiltering run-off and rainfall from the outcrop zones in the eastern region. The shallow aquifers are vulnerable to contamination since they are exposed to direct recharge by surface flows. The artesian aquifer gave free-flowing wells in about $20 \%$ of its area of occurrence $\sim 14,767 \mathrm{~km}^{2}$ [83]. The Gwandu aquifer showed artesian flows in places with heads varying from a few meters to over $25 \mathrm{~m}$ (e.g., Borehole GSN 3056 at Kurdula) and free flows up to 12,000 gph (borehole GSN 3069 at Karfin Sarki). Also, artesian flows have been encountered at Masallaci. Aquifer tests conducted in boreholes tapping the Gwandu artesian aquifer show a wide range in transmissivity. The lowest values, of less than $1000 \mathrm{gpd} / \mathrm{ft}$ at borehole GSN 2674 in Bacaka, commonly illustrate the downdip zones near the Niger boundary, such as at Kurdula and Bacaka, in Northern Kebbi State [13] (Fig. 3).

The Gwandu artesian aquifer, covering an area of about $9653 \mathrm{~km}^{2}$, can provide flowing artesian water to boreholes in valleys totaling approximately $1693.44 \mathrm{~km}^{2}$. This occurred primarily within the River Sokoto Fadama (floodplain). The flow occurs in a low-lying area trending southwest from Masallaci through Ruawuri, Balle, and Karfin
Fig. 3 Typical lithological sections of boreholes a Gwandu formation and $\mathbf{b}$ Illo formation (a) Meters LITHOLOGY

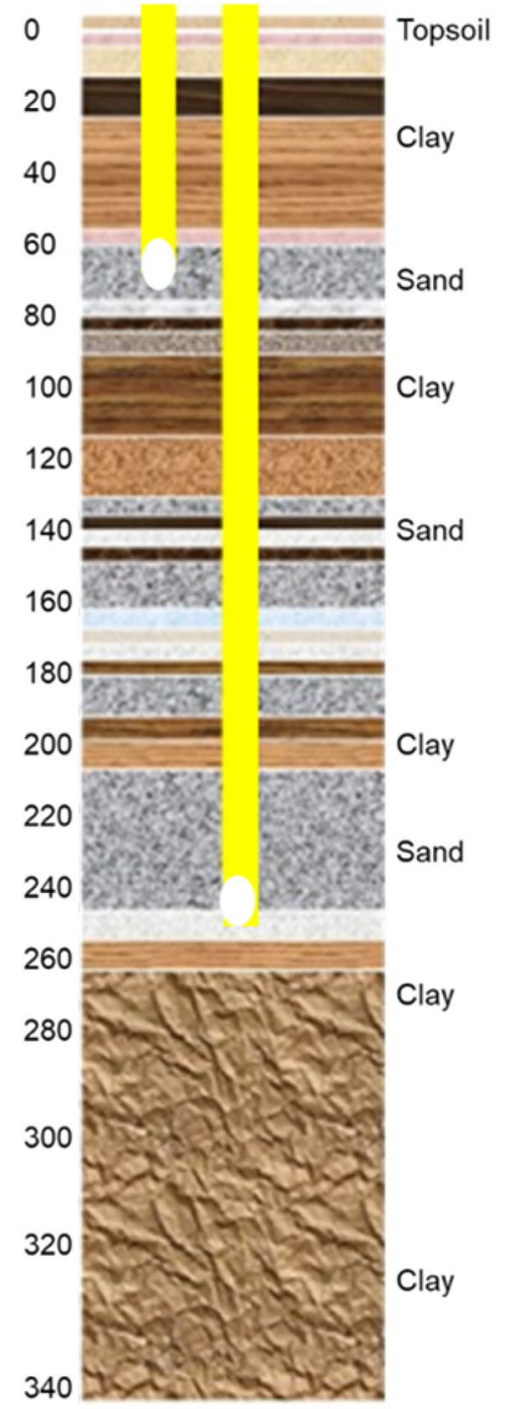

(b) Meters LITHOLOGY

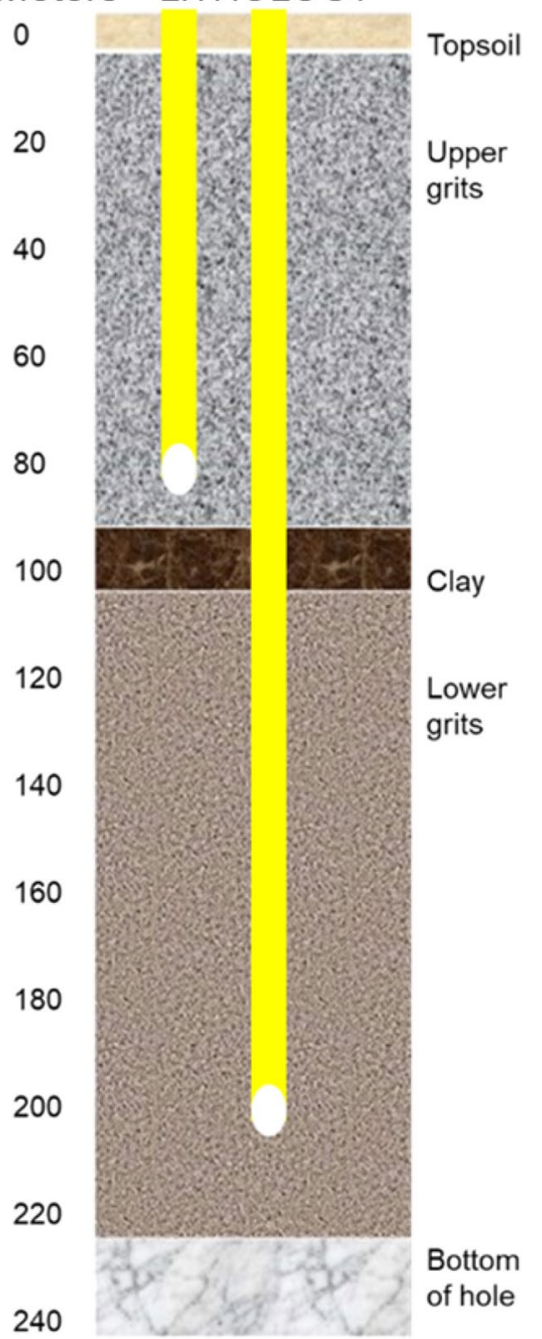


Sarki, along the Niger frontier near Kurdula and Bacaka, and in a narrow lowland stretching some $34 \mathrm{~km}$ southwest of Yeldu. With its large proven areal extent, shallow depth, and high heads, the aquifer possessed the most prominent development potential. The aquifer offers the most prominent development potential among the three artesian aquifers recognized in the Sokoto basin [13]. Concerning groundwater, the most significant part of the Gwandu formation is a sandy region in the basal section that, were outlined at depth, forms the most widespread and productive artesian aquifer hitherto known in the Sokoto basin [13]. Aquifer tests showed that artesian flows were obtained in places with heads varying from a few meters up to $25 \mathrm{~m}$ above the ground surface. Aquifer tests conducted in boreholes tapping the Gwandu artesian aquifer show a wide range in transmissivity $[5,13,46]$. Recharge into the Gwandu aquifer is predominantly by infiltration from rainfall and runoff on the outcrop zone. Groundwater extraction is chiefly by withdrawal from shallow wells and deep wells (boreholes, are on the rise and are constructed in all parts of the study area) $[6,43]$. Boreholes tapping the aquifer contribute to both the public and private water supplies at Birnin kebbi, but the estimated withdrawal was less than 100,000 gpd [13].

\subsubsection{The Illo formation}

The Illo aquifer is comprised of nonmarine cross-bedded stony clay and sand, that lie beneath an area of $\sim 4000 \mathrm{~km}^{2}$ in the southwestern Sokoto basin. The lower "grits" member of $122 \mathrm{~m}$ thick lies on Pre-Cretaceous basement crystalline rocks [13]. It is comprised of white friable medium to coarse pebbly sand with interbedded blue, yellow, red clay, and clayey sands. Very little is known about the hydrogeological character of Illo formation. Like the Gwandu aquifer, the latter has abundantly porous fine-gravel and coarse sands. In some locations, the Illo aquifer is continuous hydraulically with the Rima Group, which was characterized by an artesian aquifer, suggesting southward flow of groundwater into the Illo aquifer. Groundwater from the Illo aquifer is emptied into the floodplains areas of the Rivers Niger and Sokoto in the western Sokoto basin. Similarly, the artesian aquifer of the Gwandu formation is hydraulically continuous with the aquifers of the Rima-Illo Group. West of River Sokoto, about 32-48 km northward, the Dange formation which is a confining layer is absent. The hydrogeological conditions of these aquifers have been obstructed by human activities, owing to increased water demands by industry, agriculture, and municipal supplies.

The Illo Group is comprised of nonmarine cross-bedded pebbly sand and clay that lie beneath an area of about $6437.2 \mathrm{~km}^{2}$ in the southwestern Sokoto basin. There is no comprehensive detail on groundwater conditions in the Illo formation. The previous investigation shows that it is an extremely porous coarse sand and fine gravel aquifer, under sub-artesian environments and containing groundwater of good quality [13]. At Birnin kebbi the Illo aquifer seems to be hydraulically continuous with the artesian aquifer in the Rima group. The potentiometry analysis implied that water moves southward from the Rima aquifer into the aquifer of the Illo Group, which in turn discharges into the lower reaches of the River Sokoto and River Niger [13]. The Illo aquifer encountered at Mungadi contained $242.01 \mathrm{~m}$ of coarse sand and fine pebbles with some clay that resembles the upper portion of the Illo group in exploratory borehole GSN 3707 [13]. This unit perhaps combined near Birnin kebbi into a corresponding thick segment (from 111.25 to $305.71 \mathrm{~m}$ in borehole GSN 2484) of fine to coarse sand of the Rima group. It was interpreted here as representing a deltaic deposit that develops finer-grained northward away from the source area. Between a depth of $268.83 \mathrm{~m}$ and bedrock at $388.92 \mathrm{~m}$, borehole GSN 3707 crossed an impenetrable unit of clay intermixed with grit that resembled either to the lower part of the Illo group or perhaps to the Gundumi formation [13].

\subsection{Land use}

Numerous trans-human herds of cattle owned by Fulani graze extensively in both the fallow farmland and uncultivated areas. Shifting cultivation was common in upland areas, but with increasing population and urbanization, it is no more practiced [1]. At locations where surface water is easily accessible, settlements have been established which are associated with permanent cultivation, along the Fadama (floodplain), both during and after the rainy season [1]. Traditional Shadoof irrigation farming is widely practiced near floodplain areas or where shallow groundwater is available. Western Sokoto basin is currently the rice basket of Nigeria, irrigation farming being the primary economic activity. The increase in irrigation farming coupled with urbanization as well as poor sewage disposal posed a threat to water quality.

Land use (LU)/land cover (LC) in the study is comprised of thirteen (13) classes: dense grassland, forest zone, open grazed grassland, grassy fallow, open grain fallow farmland, open cultivation, rice paddies, pepper farms, sugarcane farmland, scrubland, barren dryland, wetland/ floodplain, and sand lodes [39]. Forty percent (40\%) of land use in the study area is under cultivation, fallow (22\%). Noncultivable or degraded land constitutes $27 \%$, and forested areas constitute $11 \%$. Expansion of farmlands and pervasiveness of grazing are major causes of forest lost in Sokoto basin. Land use changes in the study area are 
reflection of increasing human activities and effects of climate change [39]. These changes suggest underutilization of cultivable areas which have impact on hydrology, soil, and biodiversity with grave consequences for food security and livelihood [39].

\section{Materials and methods}

\subsection{Sampling, and laboratory analysis}

Sampling wells were selected from 40 locations. Twenty (20) water samples were collected, each from Gwandu and Illo aquifers. In situ analysis of physical parameters comprising of $\mathrm{pH}$, temperature, electrical conductivity, and TDS were conducted. Water quality probes (conductivity/ TDS meter, DIST; pH Meter, pHep; and temp/salinity-meter) were used. The water quality meters were calibrated using deionized water and followed by water from the sampled boreholes. Separate groundwater samples were taken using 1 liter plastic containers. These were analyzed in the laboratory. Insulated containers were used to store water samples with controlled temperatures $<5^{\circ} \mathrm{C}$. The bottles were rinsed using deionized water and dried before water samples were taken. Groundwater water samples were filtered through a "Whatman Polycap GW encapsulated filter (POLYCARP GW 75, $0.45 \mu \mathrm{m}$, polyethersulfone)" [65].

The entire analysis was conducted within $24 \mathrm{~h}$. Consequently, preservative was not added. The cations comprising of calcium $\left(\mathrm{Ca}^{2+}\right)$, magnesium $\left(\mathrm{Mg}^{2+}\right)$, sodium $\left(\mathrm{Na}^{+}\right)$, potassium $\left(\mathrm{K}^{+}\right)$, iron $\left(\mathrm{Fe}^{3+}\right)$, zinc $\left(\mathrm{Zn}^{2+}\right)$, and copper $\left(\mathrm{Cu}^{2+}\right)$ were analyzed using atomic absorption spectrometry (ST-AAS 7000 SERIES). Nitrate $\left(\mathrm{NO}_{3}{ }^{-}\right)$and phosphates $\left(\mathrm{PO}_{4}{ }^{3-}\right)$ were analyzed using automated colorimetry $\left(\mathrm{San}^{++}\right.$automated wet chemistry analyzer-continuous flow analyzer (CFA)). Chloride and $\mathrm{HCO}_{3}{ }^{-}$were analyzed by Titration- $\mathrm{HC1} 0.02$ [65]. Sulfate was determined using iron chromatography (Metrohm: ADVANCE MODULAR IC). The entire analyses were conducted in triplicates. Results were found reproducible within the error limit of $\pm 5 \%$. The raw data matrix is presented in Table 1.

\subsection{Water quality for irrigation use}

The suitability of groundwater for irrigation was assessed employing irrigation water index notably sodium adsorption ratio (SAR) as summarized in Table 2. SAR is an operative index for the assessment of alkali and/or sodium hazard to crops [15]. The SAR values are plotted versus electrical conductivity (EC). This technique was first used by US Salinity Laboratory Staff [105]. The SAR index can be applied to rate irrigation water and evaluate its suitability for irrigation usage $[108,109]$. This is essential since soil permeability can be lessened by $\mathrm{Na}^{+}$reaction with soil. Under wet conditions, cation exchange between $\mathrm{Mg}^{2+}$ and $\mathrm{Ca}^{2+}$ can be triggered by high $\mathrm{Na}^{+}$concentrations which subsequently reduce water and air circulations in the soil. SAR is defined:

$\mathrm{Na}^{+} / \sqrt{\mathrm{Ca}}+\mathrm{Mg} / 2$

\subsection{Rock weathering and ion exchange process}

The ion exchange process was evaluated using the Schoeller index [94]. Positive values suggest chloro-alkaline equilibrium base-exchange reaction, which occurs in groundwater aquifers where $\mathrm{K}^{+}$and $\mathrm{Na}^{+}$are exchanged with $\mathrm{Ca}^{2+}$ and $\mathrm{Mg}^{2+}$. The negative values indicate a choroalkaline disequilibrium base-exchange reaction (Table 2). However, the $\mathrm{Na} / \mathrm{Cl}$ molar ratio was also applied to study silicate weathering [75]. Values greater 1 suggest silicate weathering as the source of $\mathrm{Na}^{+}$in groundwater, in the absence of anthropogenic inputs. It is defined as:

$\mathrm{Cl}(\mathrm{Na}+\mathrm{K}) / \mathrm{Cl}$

\subsubsection{Geochemical modeling}

Examining the thermodynamic mechanisms on the hydrochemical composition of aquifers and calculation of equilibrium species is carried out using PHREEQC $[2,14,20,22$, 27]. Results offered saturation indices of rock minerals. The saturation index of a mineral can be computed as:

$\mathrm{SI}=\log _{10}\left(\frac{\mathrm{IAP}}{K_{\mathrm{T}}}\right)$

where the effect of ion activity of the water rock-mineral reaction is IAP, and the constant of thermodynamic equilibrium corrected to water (sample) temperature is $K_{\mathrm{T}}$. The saturation index (SI) was computed using PHREEQC interactive, version 3.5.0-14000. Table 3 summarizes the saturation indices of rock minerals.

\subsection{Statistical analysis}

\subsubsection{Correlations and analysis of variance}

Pearson's correlation analysis was applied to study the relationship between hydrochemical parameters. Several sample tests ANOVA (i.e., Kruskal-Wallis test) were applied to test the variability of hydrochemistry of the two geologic environments (Tables 3, 4). The Kruskal-Wallis test makes available for assessing numerous autonomous random water samples. It is used as a nonparametric substitute 
Table 1 Physicochemical composition of groundwater in Gwandu and Illo Geologic formations, Western Sokoto Basin

\begin{tabular}{|c|c|c|c|c|c|c|c|c|c|c|c|c|c|c|c|c|c|c|}
\hline $\begin{array}{l}\text { Sam- } \\
\text { pling } \\
\text { location }\end{array}$ & Temp & $\mathrm{EC}$ & $\mathrm{pH}$ & TDS & $\mathrm{TH}$ & $\mathrm{K}^{+}$ & $\mathrm{Na}^{+}$ & $\mathrm{Ca}^{2+}$ & $\mathrm{Cu}^{2+}$ & $\mathrm{Fe}^{3+}$ & $\mathrm{Zn}^{2+}$ & $\mathrm{Mg}^{2+}$ & $\mathrm{Cl}^{-}$ & $\mathrm{CO}_{3}$ & $\mathrm{HCO}_{3}$ & $\mathrm{PO}_{4}{ }^{3-}$ & $\mathrm{NO}_{3}^{-}$ & $\mathrm{SO}_{4}{ }^{2-}$ \\
\hline \multicolumn{19}{|c|}{ Gwandu formation } \\
\hline Sp1 & 34.0 & 80.0 & 6.7 & 40.0 & 46.5 & 37.6 & 6.7 & 17.0 & 0.3 & 1.1 & 0.4 & 1.0 & 3.7 & 0.3 & 20.0 & 1.5 & 3.1 & 31.5 \\
\hline p2 & 31.0 & 100.0 & 7.5 & 40.0 & 33.6 & 34.6 & 9.8 & 6.9 & 0.3 & 1.2 & 0.8 & 4.0 & 3.6 & 0.3 & 17.6 & 0.2 & 3.0 & 29.7 \\
\hline p3 & 31.0 & 120.0 & 7.0 & 60.0 & 50.2 & 40.3 & 0.1 & 13.5 & 0.4 & 0.9 & 0.4 & 4.0 & 4.2 & 0.2 & 16.7 & 0.2 & 2.9 & 43.2 \\
\hline p4 & 32.0 & 100.0 & 6.8 & 50.0 & 275.0 & 38.4 & 2.9 & 98.3 & 0.3 & 1.3 & 0.4 & 7.1 & 2.1 & 0.4 & 28.1 & 0.5 & 4.5 & 45.7 \\
\hline p5 & 32.0 & 60.0 & 6.9 & 30.0 & 41.2 & 34.6 & 3.9 & 3.0 & 0.3 & 0.3 & 0.2 & 8.2 & 3.6 & 0.4 & 26.0 & 1.7 & 4.2 & 52.5 \\
\hline p6 & 32.0 & 80.0 & 6.9 & 40.0 & 74.8 & 40.3 & 2.9 & 20.5 & 0.5 & 0.3 & 0.4 & 5.7 & 0.9 & 0.5 & 30.9 & 0.6 & 3.8 & 49.8 \\
\hline p7 & 34.0 & 250.0 & 7.0 & 120.0 & 66.4 & 35.3 & 13.5 & 20.0 & 0.4 & 0.5 & 0.4 & 4.0 & 2.8 & 0.0 & 2.8 & 0.3 & 5.2 & 64.4 \\
\hline p8 & 33.0 & 3160.0 & 7.0 & 1580.0 & 71.8 & 39.9 & 0.9 & 7.9 & 0.4 & 0.2 & 0.4 & 12.7 & 2.5 & 0.1 & 7.0 & 0.4 & 7.1 & 59.3 \\
\hline Sp9 & 33.0 & 8070.0 & 6.8 & 4090.0 & 138.0 & 28.5 & 9.8 & 20.6 & 0.5 & 1.0 & 0.4 & 21.1 & 1.1 & 0.2 & 13.1 & 0.8 & 6.8 & 47.6 \\
\hline Sp10 & 31.0 & 2330.0 & 8.0 & 1160.0 & 36.3 & 24.3 & 1.5 & 2.8 & 0.3 & 0.4 & 0.5 & 7.1 & 3.7 & 0.4 & 29.0 & 0.5 & 5.6 & 47.5 \\
\hline Sp11 & 32.0 & 1630.0 & 6.4 & 810.0 & 77.3 & 35.3 & 0.9 & 10.1 & 0.4 & 0.9 & 0.7 & 12.7 & 2.0 & 0.1 & 12.2 & 0.6 & 5.1 & 48.9 \\
\hline p12 & 32.0 & 1560.0 & 6.3 & 780.0 & 62.2 & 38.8 & 0.1 & 21.1 & 0.4 & 3.4 & 0.5 & 2.3 & 3.4 & 0.5 & 39.3 & 0.2 & 6.9 & 46.3 \\
\hline p13 & 33.0 & 2060.0 & 8.1 & 1050.0 & 61.6 & 22.0 & 9.8 & 18.4 & 0.3 & 1.4 & 0.4 & 3.8 & 2.9 & 0.4 & 29.3 & 3.4 & 8.2 & 52.1 \\
\hline Sp14 & 32.0 & 2100.0 & 8.0 & 1020.0 & 85.7 & 39.5 & 10.9 & 5.7 & 0.4 & 0.2 & 0.4 & 17.4 & 1.9 & 0.0 & 1.6 & 0.5 & 4.8 & 56.9 \\
\hline p15 & 32.0 & 2480.0 & 6.0 & 1230.0 & 187.4 & 39.9 & 1.5 & 43.8 & 0.6 & 7.5 & 0.6 & 19.0 & 3.4 & 0.2 & 15.2 & 0.6 & 3.4 & 48.5 \\
\hline p16 & 32.0 & 2560.0 & 5.9 & 1280.0 & 84.3 & 40.3 & 3.9 & 16.0 & 0.4 & 1.7 & 0.5 & 10.8 & 3.8 & 0.6 & 40.0 & 0.8 & 6.3 & 50.1 \\
\hline p17 & 31.0 & 2310.0 & 6.3 & 1150.0 & 93.9 & 32.7 & 1.5 & 20.5 & 0.4 & 0.5 & 0.5 & 10.4 & 2.9 & 0.3 & 20.7 & 0.5 & 4.9 & 48.1 \\
\hline p18 & 31.0 & 2050.0 & 6.3 & 1010.0 & 25.7 & 37.2 & 9.8 & 0.3 & 0.3 & 2.2 & 0.3 & 6.1 & 3.3 & 0.1 & 4.7 & 0.3 & 5.6 & 39.5 \\
\hline Sp19 & 33.0 & 2670.0 & 6.1 & 1340.0 & 62.2 & 35.0 & 0.1 & 11.4 & 0.3 & 2.6 & 0.3 & 8.2 & 3.3 & 0.4 & 27.5 & 0.4 & 7.4 & 40.2 \\
\hline Sp20 & 33.0 & 2710.0 & 6.1 & 1350.0 & 84.2 & 31.9 & 12.8 & 5.2 & 0.1 & 1.3 & 0.5 & 17.4 & 1.5 & 0.1 & 4.1 & 0.1 & 8.1 & 45.3 \\
\hline \multicolumn{19}{|c|}{ Illo formation } \\
\hline Sp1 & & 20.0 & 1.6 & 30.0 & 20.7 & 39.0 & 299.0 & 5.0 & 1.1 & 0.6 & 0.1 & 2.0 & 36.0 & 2.7 & 183.0 & 67.0 & 12.5 & 23.5 \\
\hline p2 & 31.1 & 570.0 & 2.9 & 640.0 & 132.3 & 234.0 & 437.0 & 48.0 & 1.0 & 0.5 & 6.3 & 3.0 & 107.0 & 6.3 & 427.0 & 16.0 & 11.7 & 67.4 \\
\hline p3 & 31.4 & 0.0 & 1.7 & 70.0 & 24.8 & 39.0 & 460.0 & 5.0 & 0.8 & 2.3 & 6.8 & 3.0 & 142.0 & 1.8 & 122.0 & 20.0 & 13.1 & 90.7 \\
\hline p4 & 32.1 & 180.0 & 1.6 & 320.0 & 62.1 & 39.0 & 460.0 & 15.0 & 0.5 & 1.2 & 5.3 & 6.0 & 213.0 & 1.8 & 122.0 & 24.0 & 14.0 & 110.3 \\
\hline p5 & 31.2 & 100.0 & 1.7 & 140.0 & 62.1 & 39.0 & 460.0 & 15.0 & 0.5 & 1.2 & 5.3 & 6.0 & 213.0 & 2.8 & 122.0 & 24.0 & 12.9 & 90.9 \\
\hline p6 & 30.6 & 250.0 & 1.4 & 250.0 & 116.0 & 156.0 & 2277.0 & 30.0 & 1.1 & 0.3 & 6.3 & 10.0 & 3444.0 & 0.9 & 61.0 & 17.0 & 13.5 & 87.7 \\
\hline sp7 & 29.6 & 40.0 & 1.7 & 50.0 & 23.2 & 39.0 & 368.0 & 6.0 & 0.5 & 2.3 & 4.3 & 2.0 & 36.0 & 3.6 & 244.0 & 12.0 & 12.6 & 46.1 \\
\hline $5 p 8$ & 29.3 & 50.0 & 1.5 & 50.0 & 69.2 & 39.0 & 414.0 & 8.0 & 0.2 & 0.8 & 5.2 & 12.0 & 142.0 & 1.8 & 122.0 & 13.0 & 11.8 & 70.5 \\
\hline $5 \mathrm{p} 9$ & 30.3 & 10.0 & 1.9 & 10.0 & 404.8 & 273.0 & 184.0 & 157.0 & 0.3 & 1.8 & 4.2 & 3.0 & 6639.0 & 5.4 & 366.0 & 30.0 & 10.5 & 88.4 \\
\hline p10 & 30.6 & 130.0 & 3.0 & 160.0 & 102.3 & 39.0 & 345.0 & 36.0 & 1.0 & 1.5 & 3.4 & 3.0 & 36.0 & 3.6 & 244.0 & 22.0 & 12.3 & 78.2 \\
\hline Sp11 & 24.6 & 100.0 & 2.5 & 150.0 & 542.0 & 78.0 & 2162.0 & 20.0 & 1.8 & 2.3 & 5.2 & 120.0 & 3408.0 & 0.9 & 51.0 & 18.0 & 5.7 & 70.8 \\
\hline Sp12 & 26.7 & 34.0 & 2.8 & 370.0 & 60.5 & 39.0 & 529.0 & 16.0 & 1.1 & 1.0 & 6.2 & 5.0 & 213.0 & 3.6 & 244.0 & 1.0 & 8.1 & 111.2 \\
\hline Sp13 & 29.1 & 140.0 & 2.8 & 150.0 & 58.9 & 39.0 & 414.0 & 17.0 & 0.5 & 1.1 & 0.7 & 4.0 & 213.0 & 1.8 & 122.0 & 36.0 & 10.3 & 98.4 \\
\hline Sp14 & 29.8 & 110.0 & 2.2 & 120.0 & 51.4 & 39.0 & 529.0 & 14.0 & 1.4 & 2.2 & 6.3 & 4.0 & 349.0 & 1.8 & 122.0 & 22.0 & 3.8 & 76.5 \\
\hline Sp15 & 30.2 & 20.0 & 8.1 & 200.0 & 22.3 & 39.0 & 483.0 & 4.0 & 1.6 & 1.6 & 6.1 & 3.0 & 142.0 & 1.8 & 122.0 & 12.0 & 7.2 & 87.1 \\
\hline Sp16 & 30.8 & 110.0 & 8.4 & 130.0 & 50.5 & 39.0 & 460.0 & 12.0 & 0.4 & 2.0 & 4.1 & 5.0 & 284.0 & 1.8 & 122.0 & 20.0 & 9.8 & 93.5 \\
\hline Sp17 & 29.4 & 80.0 & 9.2 & 910.0 & 289.8 & 39.0 & 460.0 & 111.0 & 0.4 & 2.1 & 3.2 & 3.0 & 1598.0 & 3.6 & 244.0 & 22.0 & 87.0 & 90.8 \\
\hline Sp18 & 31.1 & 40.0 & 9.0 & 70.0 & 29.1 & 39.0 & 437.0 & 10.0 & 0.6 & 0.6 & 6.3 & 1.0 & 142.0 & 6.3 & 432.0 & 26.0 & 9.1 & 112.4 \\
\hline Sp19 & 29.3 & 20.0 & 8.5 & 160.0 & 53.2 & 78.0 & 552.0 & 18.0 & 0.4 & 0.6 & 7.5 & 2.0 & 178.0 & 1.8 & 122.0 & 35.0 & 11.2 & 89.7 \\
\hline Sp20 & 29.3 & 1660.0 & 8.4 & 1970.0 & 285.5 & 156.0 & 690.0 & 106.0 & 0.4 & 1.9 & 5.7 & 5.0 & 2237.0 & 5.4 & 366.0 & 22.0 & 10.4 & 78.9 \\
\hline
\end{tabular}

The entire concentration is in milligram per liter (mg/l). Temperature $\left({ }^{\circ} \mathrm{C}\right), \mathrm{EC}(\mu \mathrm{S} / \mathrm{cm})$ and $\mathrm{pH}$ (unit)

Bold value indicate extreme concentration 
Table 2 Schoeller index (Si), molar ratio (MR), and sodium adsorption ratio (SAR)

\begin{tabular}{|c|c|c|c|c|c|c|}
\hline \multirow[t]{2}{*}{ Samples } & \multicolumn{3}{|c|}{ Gwandu formation } & \multicolumn{3}{|c|}{ Illo formation } \\
\hline & Si & MR & SAR & Si & MR & SAR \\
\hline Sp 1 & 44.33 & 1.82 & 11.20 & 338.00 & 8.31 & 300.75 \\
\hline Sp 2 & 44.38 & 2.73 & 12.52 & 671.00 & 4.08 & 449.75 \\
\hline Sp 3 & 40.38 & 0.02 & 4.48 & 499.00 & 3.24 & 462.00 \\
\hline Sp 4 & 41.28 & 1.39 & 29.26 & 499.00 & 2.16 & 465.25 \\
\hline Sp 5 & 38.46 & 1.07 & 6.68 & 499.00 & 2.16 & 465.25 \\
\hline Sp 6 & 43.18 & 3.19 & 9.46 & 2433.00 & 0.66 & 2287.00 \\
\hline Sp 7 & 48.79 & 4.82 & 19.45 & 407.00 & 10.22 & 370.00 \\
\hline Sp 8 & 40.81 & 0.36 & 6.06 & 453.00 & 2.92 & 419.00 \\
\hline Sp 9 & 38.30 & 9.25 & 20.23 & 457.00 & 0.03 & 224.00 \\
\hline Sp 10 & 25.83 & 0.41 & 4.00 & 384.00 & 9.58 & 354.75 \\
\hline Sp 11 & 36.25 & 0.46 & 6.61 & 2240.00 & 0.63 & 2197.00 \\
\hline Sp 12 & 38.86 & 0.03 & 5.95 & 568.00 & 2.48 & 534.25 \\
\hline Sp 13 & 31.85 & 3.37 & 15.36 & 453.00 & 1.94 & 419.25 \\
\hline Sp 14 & 50.46 & 5.76 & 16.72 & 568.00 & 1.52 & 533.50 \\
\hline Sp 15 & 41.41 & 0.44 & 17.21 & 522.00 & 3.40 & 484.75 \\
\hline Sp 16 & 44.18 & 1.02 & 10.60 & 499.00 & 1.62 & 464.25 \\
\hline Sp 17 & 34.19 & 0.52 & 9.24 & 499.00 & 0.29 & 488.50 \\
\hline Sp 18 & 47.04 & 3.01 & 11.40 & 476.00 & 3.08 & 439.75 \\
\hline Sp 19 & 35.06 & 0.03 & 5.01 & 630.00 & 3.10 & 557.00 \\
\hline Sp 20 & 44.72 & 8.71 & 18.44 & 846.00 & 0.31 & 717.75 \\
\hline Min & 25.83 & 0.02 & 4.00 & 338.00 & 0.03 & 224.00 \\
\hline Max & 50.46 & 9.25 & 29.26 & 2433.00 & 10.22 & 2287.00 \\
\hline Mean & 40.28 & 2.62 & 12.42 & 759.64 & 3.27 & 688.40 \\
\hline SE & 9.01 & 0.59 & 2.78 & 169.86 & 0.73 & 153.93 \\
\hline
\end{tabular}

for the one-way ANOVA. The measurement for $k$ samples, for each of size $n_{i}$, is computed:

$T=\frac{1}{S^{2}}\left[\sum \frac{k}{i=1} \frac{R_{i}}{n_{i}}-N \frac{(N+1)^{2}}{4}\right]$

where $N=$ sum of all observations (all $n_{i}$ ) and $R_{i}=$ total of the ranks (of the entire samples taken) for the ith sample and:

$S^{2} \frac{1}{N-1}=\left[\sum_{\text {all }} R_{i j}^{2}-N \frac{(N+1)^{2}}{4}\right]$

The $H_{o}$ of the analysis assumed that the entire $k$ distribution functions are the same. The $H_{1}$ assumed that one of the observations tends to produce a bigger score over at least one of the other observations $[21,70]$.

\subsubsection{Factor analysis}

Analysis of hydrochemical data is a multivariate problem. Factor analysis (FA) was used to examine the interconnections among the hydrochemical variables. Factor analysis classifies enormous and convoluted datasets by defining an insignificant number of variables that illuminate for ultimate variance within an original set of data. Factor analysis was conducted on a subset of 18 chosen elements (temperature, $\mathrm{EC}, \mathrm{pH}, \mathrm{TDS}, \mathrm{TH}, \mathrm{K}^{+}, \mathrm{Na}^{+}, \mathrm{Ca}^{2+}, \mathrm{Cu}^{2+}, \mathrm{Fe}^{3+}, \mathrm{Zn}^{2+}$, $\left.\mathrm{Mg}^{2+}, \mathrm{Cl}^{-}, \mathrm{HCO}_{3}{ }^{-}, \mathrm{CO}_{3}{ }^{2-}, \mathrm{PO}_{4}{ }^{3-}, \mathrm{NO}_{3}, \mathrm{SO}_{4}{ }^{2-}\right)$ as contained in Table 1.

\subsubsection{Hierarchical cluster analysis}

Hierarchical cluster analysis (HCA) was applied to gain ultimate insight of hydrochemistry of groundwater by classifying the sampled boreholes into distinct clusters [16, $19,65]$. This technique of clustering is beneficial for not needing any prior hypothesis of the clustering or number of clusters. In grouping, boreholes having similar hydrochemistry or else boreholes having different hydrochemical properties cluster into distinguishable collections. The Ward's-algorithmic technique was used. It is deemed as a very strong tool for clustering. The technique is capable of curbing the perverting influence or sum of squared distances of centroids between two theoretical groupings created at each step. Therefore, applying factor scores into HCA is an outstanding procedure for analyzing data by simplifying groups into a more communicative manner, 
Table 3 Summary of SIs derived from geochemical modeling with PHREEQC

\begin{tabular}{|c|c|c|c|c|c|c|c|c|c|c|c|}
\hline \multirow[t]{2}{*}{ S/no } & \multirow[t]{2}{*}{ Mineral phases } & \multicolumn{4}{|c|}{ Gwandu formation } & \multicolumn{4}{|c|}{ Illo formation } & \multicolumn{2}{|c|}{ Kruskal-Wallis test } \\
\hline & & Min & Max & Mean & SE & Min & Max & Mean & SE & $H$ (chi2) & $P$ (same) \\
\hline 1 & $\mathrm{Al}(\mathrm{OH}) 3(\mathrm{a})$ & -3.12 & -2.62 & -2.911 & -0.67 & -3.14 & -2.64 & -2.936 & -0.70 & 3.187 & 0.073 \\
\hline 2 & Albite & -1.53 & -0.24 & -1.338 & -0.33 & -1.5 & -1.22 & -1.423 & -0.28 & 0.896 & 0.34 \\
\hline 3 & Alunite & -3 & 2.8 & 1.52 & 0.61 & 0.97 & 4.29 & 2.485 & 0.56 & 5.666 & 0.017 \\
\hline 4 & Anhydrite & -0.02 & 0 & -0.002 & 0.00 & -0.01 & 0 & -0.001 & 0.00 & 0 & 0.971 \\
\hline 5 & Anorthite & -7.76 & -3.8 & -6.773 & -1.70 & -8.2 & -5.86 & -7.346 & -1.76 & 9.015 & 0.003 \\
\hline 6 & Aragonite & -0.14 & -0.14 & -0.14 & -0.03 & -0.14 & -0.14 & -0.14 & -0.03 & 0 & 0.971 \\
\hline 7 & Ca-montmorillonite & -2.92 & -2.26 & -2.711 & -0.60 & -2.98 & -2.15 & -2.725 & -0.59 & 2.59 & 0.104 \\
\hline 8 & Calcite & 0 & 0 & 0 & 0.00 & 0 & 0 & 0 & 0.00 & 0 & 0.971 \\
\hline 9 & $\mathrm{CH} 4(\mathrm{~g})$ & -14.64 & -6.7 & -12.789 & -3.05 & -14.4 & -13.66 & -13.959 & -3.11 & 6.534 & 0.01 \\
\hline 10 & Chalcedony & -0.89 & -0.4 & -0.609 & -0.11 & -0.9 & -0.41 & -0.598 & -0.09 & 0.143 & 0.695 \\
\hline 11 & Chlorite(14A) & -16.33 & 2.88 & -11.673 & -3.56 & -17.62 & -7.16 & -14.203 & -3.71 & 10.54 & 0.001 \\
\hline 12 & Chrysotile & -11.38 & -0.45 & -8.782 & -2.51 & -12.23 & -6.43 & -10.238 & -2.55 & 8.067 & 0.004 \\
\hline 13 & $\mathrm{CO}_{2}(\mathrm{~g})$ & -3.84 & 0 & -0.906 & 0.00 & -1.94 & 0 & -0.508 & 0.00 & 1.029 & 0.211 \\
\hline 14 & Dolomite & 0 & 0 & 0 & 0.00 & 0 & 0 & 0 & 0.00 & 0 & 0.971 \\
\hline 15 & $\mathrm{Fe}(\mathrm{OH}) 3(\mathrm{a})$ & -14.98 & 0.14 & -3.459 & -0.44 & -2.12 & -0.55 & -1.582 & -0.39 & 0.703 & 0.391 \\
\hline 16 & $\mathrm{FeS}(\mathrm{ppt})$ & -9.61 & -3.17 & -4.832 & -0.98 & -4.72 & -3.5 & -4.24 & -0.99 & 2.254 & 0.133 \\
\hline 17 & Gibbsite & -0.48 & 0 & -0.284 & -0.09 & -0.49 & 0 & -0.288 & -0.11 & 0.053 & 0.808 \\
\hline 18 & Goethite & -8.72 & 6.35 & 2.799 & 0.97 & 3.91 & 5.66 & 4.593 & 0.99 & 1.23 & 0.267 \\
\hline 19 & Gypsum & -0.04 & 0 & -0.014 & -0.01 & -0.02 & 0 & -0.004 & 0.00 & 6.193 & 0.007 \\
\hline 20 & $\mathrm{H} 2(\mathrm{~g})$ & -9.78 & -7.9 & -9.311 & -2.17 & -10.08 & -9.47 & -9.77 & -2.21 & 13.04 & 0 \\
\hline 21 & $\mathrm{H} 2 \mathrm{O}(\mathrm{g})$ & -1.48 & -1.39 & -1.44 & -0.32 & -1.67 & -1.45 & -1.521 & -0.34 & 23.58 & 0 \\
\hline 22 & $\mathrm{H} 2 \mathrm{~S}(\mathrm{~g})$ & -12.65 & -4.71 & -10.795 & -2.60 & -12.36 & -11.66 & -11.923 & -2.66 & 5.927 & 0.015 \\
\hline 23 & Halite & -0.74 & 0 & -0.377 & -0.12 & -0.62 & 0 & -0.341 & -0.11 & 2.094 & 0.145 \\
\hline 24 & Hematite & -15.29 & 14.86 & 7.758 & 2.42 & 9.98 & 13.47 & 11.342 & 2.46 & 1.291 & 0.256 \\
\hline 25 & Illite & -1.76 & -0.8 & -1.53 & -0.39 & -1.8 & -1.26 & -1.62 & -0.40 & 8.221 & 0.004 \\
\hline 26 & Jarosite-K & -42.05 & -1.25 & -8.91 & -0.64 & -3.97 & -1.85 & -2.785 & -0.59 & 0.797 & 0.372 \\
\hline 27 & K-feldspar & 0 & 0 & 0 & 0.00 & 0 & 0 & 0 & 0.00 & 0 & 0.971 \\
\hline 28 & K-mica & 4.91 & 5.88 & 5.319 & 1.14 & 4.91 & 5.92 & 5.318 & 1.10 & 0.002 & 0.968 \\
\hline 29 & Kaolinite & 0 & 0 & 0 & 0.00 & 0 & 0.64 & 0.058 & 0.00 & 0 & 0.971 \\
\hline 30 & Mackinawite & -8.88 & -2.43 & -4.1 & -0.81 & -3.99 & -2.77 & -3.506 & -0.82 & 2.254 & 0.133 \\
\hline 31 & Melanterite & -13.62 & -1.22 & -3.597 & -0.34 & -1.86 & -1.23 & -1.573 & -0.29 & 2.055 & 0.148 \\
\hline 32 & $\mathrm{O}_{2}(\mathrm{~g})$ & -65.26 & -61.26 & -62.536 & -13.76 & -63.58 & -61.76 & -62.52 & -13.94 & 2.377 & 0.123 \\
\hline 33 & Pyrite & 0 & 0 & 0 & 0.00 & 0 & 0 & 0 & 0.00 & 0 & 0.971 \\
\hline 34 & Quartz & -0.48 & 0 & -0.2 & -0.02 & -0.49 & 0 & -0.184 & 0.00 & 0.026 & 0.869 \\
\hline 35 & Sepiolite & -9.11 & -2.52 & -7.606 & -2.03 & -9.68 & -6.46 & -8.49 & -2.00 & 6.126 & 0.013 \\
\hline 36 & Sepiolite(d) & -12.19 & -5.62 & -10.692 & -2.73 & -12.57 & -9.49 & -11.501 & -2.67 & 0.975 & 0.32 \\
\hline 37 & Siderite & -8.17 & 4.22 & 1.876 & 0.89 & 3.61 & 4.2 & 3.911 & 0.93 & 0.097 & 0.754 \\
\hline 38 & $\mathrm{SiO}_{2}(\mathrm{a})$ & -1.71 & -1.21 & -1.426 & -0.30 & -1.73 & -1.23 & -1.423 & -0.28 & 0.574 & 0.422 \\
\hline 39 & Smithsonite & -0.28 & 3.56 & 2.542 & 0.76 & 1.59 & 3.54 & 2.926 & 0.79 & 0.354 & 0.55 \\
\hline 40 & Sphalerite & 2.59 & 10.19 & 4.321 & 0.77 & 2.93 & 3.44 & 3.271 & 0.77 & 2.813 & 0.092 \\
\hline 41 & Sulfur & -9.11 & -2.63 & -7.403 & -1.75 & -8.79 & -7.8 & -8.099 & -1.77 & 0.003 & 0.955 \\
\hline 42 & Sylvite & -1.61 & -0.48 & -0.858 & -0.20 & -1.2 & -0.36 & -0.781 & -0.26 & 29.27 & 0 \\
\hline 43 & Talc & -8.38 & 1.69 & -4.713 & 0.00 & -8.49 & -8.49 & -8.49 & 0.00 & -21.37 & 1 \\
\hline 44 & Willemite & 3.85 & 4.75 & 4.19 & 0.95 & 3.21 & 4.51 & 3.976 & 0.97 & 5.287 & 0.021 \\
\hline 45 & $\mathrm{Zn}(\mathrm{OH}) 2(\mathrm{e})$ & -0.34 & 0 & -0.119 & -0.04 & -0.29 & 0 & -0.113 & 0.00 & 0.124 & 0.705 \\
\hline
\end{tabular}

Values in bold indicating the supersaturation and/or equilibrium of minerals show a significant difference between Gwandu and Illo Formations 
Table 4 Physicochemical parameters of groundwater (bold values did not follow The NSDWQ and WHO reference guidelines)

\begin{tabular}{|c|c|c|c|c|c|c|c|c|c|c|c|c|}
\hline \multirow[t]{2}{*}{ Parameters } & \multicolumn{4}{|c|}{ Gwandu formation } & \multicolumn{4}{|c|}{ Illo formation } & \multicolumn{2}{|c|}{ Kruskal-Wallis test } & \multicolumn{2}{|c|}{ Reference guidelines } \\
\hline & Min & Max & Mean & SE & Min & Max & Mean & SE & $H\left(\mathrm{chi}^{2}\right)$ & $P$ (same) & WHO (2011) & NSDWQ (2007) \\
\hline Temp & 31.0 & 34.0 & 32.2 & 7.2 & 24.6 & 32.1 & 29.8 & 6.7 & 17.35 & $<0.001^{* *}$ & - & - \\
\hline EC & 60.0 & 8070.0 & 2027.7 & 453.4 & 10.0 & 1660.0 & 244.7 & 54.7 & 14.24 & $<0.001^{* *}$ & 1000 & 1000 \\
\hline $\mathrm{pH}$ & 5.9 & 8.1 & 6.8 & 1.5 & 1.4 & 9.2 & 4.2 & 0.9 & 4.801 & $0.028^{* *}$ & 8.5 & 8.5 \\
\hline TDS & 30.0 & 4090.0 & 1015.9 & 227.2 & 10.0 & 1970.0 & 360.5 & 80.6 & 3.285 & 0.069 & 500 & 500 \\
\hline TH & 0.3 & 105.0 & 33.5 & 7.5 & 1.0 & 485.2 & 182.5 & 40.8 & 0.143 & 0.704 & 200 & 150 \\
\hline $\mathrm{K}^{+}$ & 22.0 & 40.3 & 34.9 & 7.8 & 39.0 & 273.0 & 83.3 & 18.6 & 9.846 & $<0.001^{* *}$ & - & - \\
\hline $\mathrm{Na}^{+}$ & 0.1 & 13.5 & 5.3 & 1.2 & 184.0 & 2277.0 & 676.4 & 151.2 & 29.27 & $<0.001^{* *}$ & 12 & 12 \\
\hline $\mathrm{Ca}^{2+}$ & 0.3 & 98.3 & 21.0 & 4.7 & 4.0 & 157.0 & 37.0 & 8.3 & 1.398 & 0.236 & 500 & 500 \\
\hline $\mathrm{Cu}^{2+}$ & 0.1 & 0.6 & 0.4 & 0.1 & 0.2 & 1.8 & 0.8 & 0.2 & 12.05 & $<0.001^{*}$ & 1.0 & 1.0 \\
\hline $\mathrm{Fe}^{3+}$ & 0.2 & 7.5 & 1.7 & 0.4 & 0.3 & 2.3 & 1.4 & 0.3 & 1.253 & 0.262 & 2 & 1.0 \\
\hline $\mathrm{Zn}^{2+}$ & 0.2 & 0.8 & 0.4 & 0.1 & 0.1 & 7.5 & 4.8 & 1.1 & 23.45 & $<0.001^{* *}$ & 3.0 & 3.0 \\
\hline $\mathrm{Mg}^{2+}$ & 1.0 & 21.1 & 9.3 & 2.1 & 1.0 & 120.0 & 14.7 & 3.3 & 5.298 & $0.020^{*}$ & - & $50^{*}$ \\
\hline $\mathrm{Cl}^{-}$ & 0.9 & 4.2 & 2.8 & 0.6 & 36.0 & 6639.0 & 1202.1 & 268.8 & 30.77 & $<0.001^{* *}$ & 200 & 200 \\
\hline $\mathrm{HCO}_{3}^{-}$ & 1.6 & 40.0 & 19.4 & 4.3 & 61.0 & 432.0 & 202.9 & 45.4 & 26.46 & $<0.001^{* *}$ & 250 & 250 \\
\hline $\mathrm{CO}_{3}{ }^{2-}$ & 0.0 & 0.6 & 0.3 & 0.1 & 0.9 & 6.3 & 3.0 & 0.7 & 30.77 & $<0.001^{* *}$ & - & - \\
\hline $\mathrm{PO}_{4}^{3-}$ & 0.1 & 3.4 & 0.8 & 0.2 & 1.0 & 67.0 & 24.0 & 5.4 & 29.66 & $<0.001^{* *}$ & 0.2 & 0.2 \\
\hline $\mathrm{NO}_{3}^{-}$ & 2.9 & 8.2 & 5.4 & 1.2 & 3.8 & 87.0 & 17.2 & 3.8 & 23.45 & $<0.001^{* *}$ & 50 & 50 \\
\hline $\mathrm{SO}_{4}^{2-}$ & 29.7 & 64.4 & 47.3 & 10.6 & 23.5 & 112.4 & 81.8 & 18.3 & 22.25 & $<0.001^{* *}$ & 200 & 100 \\
\hline
\end{tabular}

The entire ion concentrations are in milligram per liter $(\mathrm{mg} / \mathrm{l})$. Temperature $\left({ }^{\circ} \mathrm{C}\right), \mathrm{EC}(\mu \mathrm{S} / \mathrm{cm})$, and $\mathrm{pH} @ 25^{\circ} \mathrm{C}$

*Significant value $p=<0.05,{ }^{*}$ Significant level $p=<0.001$

despite the unfamiliarity of some hypothetically significant hydrochemical data. However, this technique does not account for the authenticity of hydrochemical data and disconnected values. There are neglects of credible and possible connections or resemblances (statistical interference) between variables that are somewhat clear in most hydrochemical data. As a result, the output would have been inevitable to manipulation and not be considered as complete hydrochemically meaningful data. Therefore, only raw data were included in the HCA. It enables a thorough examination of each grouping and real condition (i.e., no statistical interfering) of hydrochemical data can be achieved [65].

\section{Results and discussion}

The hydrochemical composition of groundwater in Gwandu and Illo formations is summarized in Table 4. The physical parameters (Temperature, EC, and TDS) showed a significant difference between geological formations. However, hardness did not correspond to geological variability. The temperature level was generally high in both Gwandu and Illo aquifers $\left(\bar{x}>25^{\circ} \mathrm{C}\right)$. Water temperature tends to be closely associated with EC; the later rises by $2 \%$ with an elevated temperature level of $1{ }^{\circ} \mathrm{C}[107,109]$. Temperature erraticism between 5 and $10^{\circ} \mathrm{C}$ in gravity flow water affects TDS levels, which eventually disturbs $\mathrm{pH}$ level, speciation, redox reaction, complexation, sorption activities, and solubility of gasses. To a groundwater analyst, EC is a parameter of considerable importance, since it is often related to the ionic content of water which informs the range into which ions concentration in water is likely to fall.

Groundwater is more acidic in the Illo aquifer ( $\bar{x}$ $\mathrm{pH} \geq 4.2$ ). In the eastern section of the Sokoto basin and the south, the most acidic water was discovered [13]. Aquifers in this area are characterized by a low TDS level $(28-79 \mathrm{mg} / \mathrm{l})$. Sulfate is the major anion, which is derived from pyrite mineral oxidation [13]. This process accounted for the acidity of the water in the basin. The result obtained from geochemical modeling (saturation indices) showed the undersaturation of pyrite minerals in both Gwandu and Illo aquifers (Table 3 ). The variability of physical parameters is further shown in Fig. 4.

\subsection{Classification of groundwater}

Total dissolved solids (TDS) and total hardness (TH) were used to classify water and assess its aptness for ingestion $[33,93]$. Results have shown that groundwater is suitable for drinking based on TDS (Table 5). However, classification based on EC showed $55 \%$ of groundwater samples from Gwandu aquifer fall into Doubtful-Unsuitable Class. In 

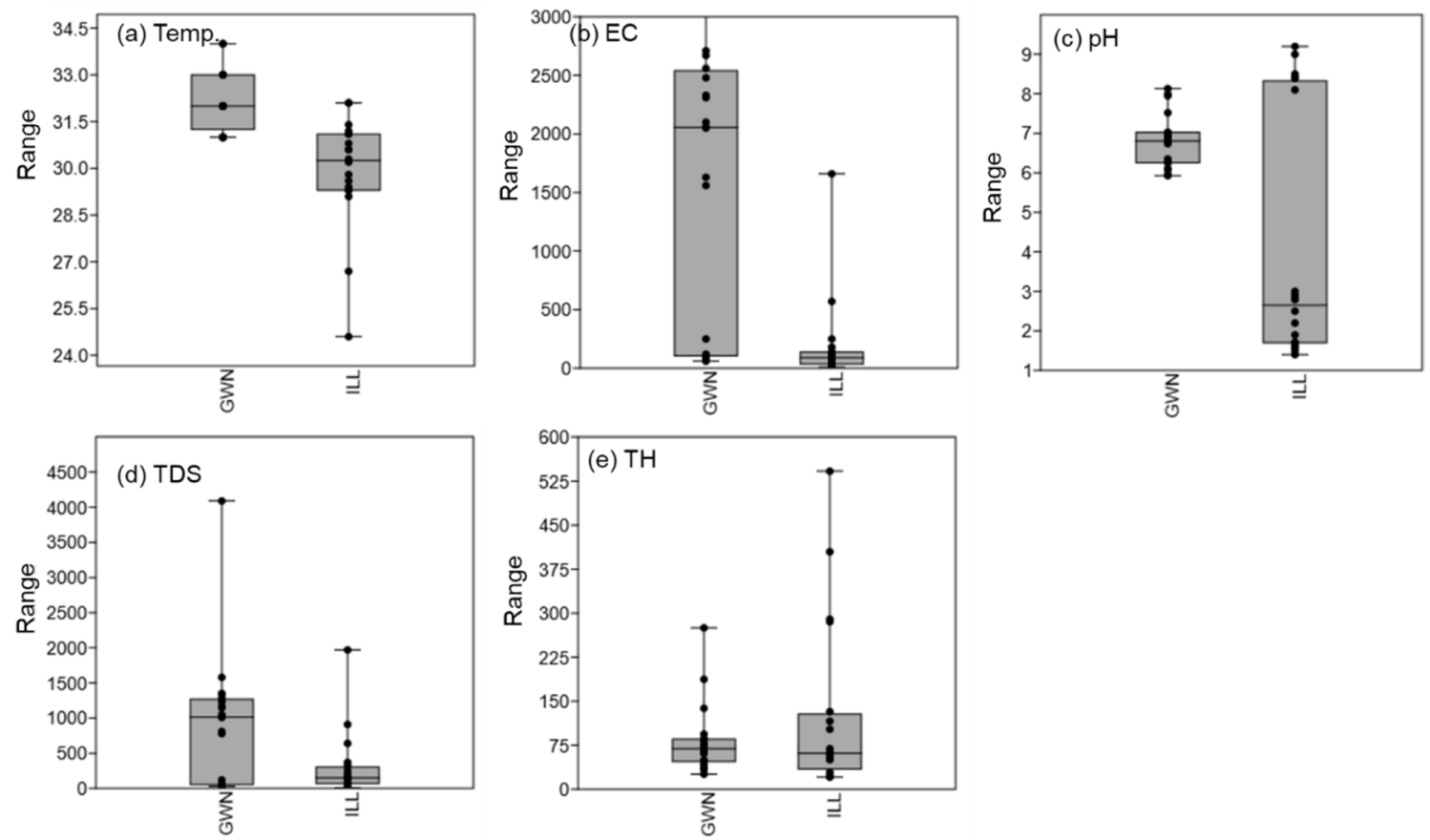

Fig. 4 Variability of physical parameters; $\mathbf{a}$ temperature; $\mathbf{b}$ electrical conductivity; $\mathbf{c} \mathbf{p H}$; $\mathbf{d}$ total dissolved solids; and $\mathbf{e}$ hardness

contrast, $95 \%$ of groundwater samples from the Illo aquifer fall into Excellent-Good Class [23]. Aquifers having the total hardness in the range of $0-75 \mathrm{mg} / \mathrm{l}$ are classified as soft [93].

However, soft waters (i.e., hardness $<80 \mathrm{mg} / \mathrm{l}$ ) tend to have a low buffering capacity and can be more corrosive to water pipes. Depending on $\mathrm{pH}$ and alkalinity, hardness above $200 \mathrm{mg} / \mathrm{l}$ can result in scale deposition, particularly on heating. Several environmental and analytical epidemiological studies have revealed a statistically significant inverse relationship between drinking hard waters and cardiovascular illness $[41,61,81]$. However, there are some indications that very soft waters may have a serious effect on mineral balance [18, 24], though detailed investigations are needed for additional evaluation [110].

\subsection{Groundwater chemistry}

Calcium and potassium were outstanding elements in Gwandu aquifer (Fig. 5). Although $\mathrm{K}^{+}$is not limited in drinking water, a higher concentration is associated with toxicity [111]. Sodium differs significantly between the two geologic environments, and like $\mathrm{K}^{+}$, it is not limited in drinking water. It is a nutritional requirement, which is monitored for its joint effect with $\mathrm{SO}_{4}{ }^{2-}$. Since drinking water having high $\mathrm{Na}^{+}$consumption can be associated with hypertension, the risk of being hypertensive is higher among people deriving drinking water from the Illo aquifer. Calcium concentration differs significantly (Table 4).
Higher concentrations of $\mathrm{Ca}^{2+}$ in drinking water have some health benefits; elevated $\mathrm{Ca}^{2+}$ and $\mathrm{Mg}^{2+}$ in aquifers are associated with hardness. Like $\mathrm{Ca}^{2+}, \mathrm{Mg}^{2+}$ is an indispensable dietary requirement $\sim 0.3-0.5 \mathrm{~g} /$ day. High ingestion is not accompanied by any adverse health threat. But some incidental health effects may be caused when considered in conjunction with sulfate $[55,89]$. The importance of $\mathrm{Ca}^{2+}$ in hydrochemical analysis relates to hardness. Calcium is found naturally in various environmental settings and occurs widely in groundwater aquifers. Dissolved calcium hydroxide forms soda and hydrogen gas. It typically occurs when $\mathrm{CO}_{2}$ is freed, resulting in the development of carbonic acid, affecting $\mathrm{Ca}^{2+}$ compounds. The significance of $\mathrm{Mg}^{2+}$ is that it constitutes a second major component of hardness $\left(\mathrm{CaCO}_{3}\right)$. Dolomite and magnesium carbonate are primary sources of $\mathrm{Mg}^{2+}$ in aquifers $[26,72,89]$. Copper and $\mathrm{Fe}^{3+}$ concentrations differ significantly. High $\mathrm{Cu}^{2+}$ ingestion is not connected to any health threat, as healing dosages of $20 \mathrm{mg} / \mathrm{l}$ are sporadically allowed. Similarly, high $\mathrm{Fe}^{3+}$ ingestion is not associated with any serious health risk. In natural waters, $\mathrm{Fe}^{3+}$ concentrations may range from 0.5 to $50 \mathrm{mg} / \mathrm{l}[53,88]$. Zinc concentration also differs significantly; in the Illo aquifer, mean $\mathrm{Zn}^{2+}$ exceeds WHO and NSDWQ reference guidelines.

Excessive intake can be associated with emetic effect and elevated levels can be toxic to aquatic animals. Unfavorable taste can be noticed at a concentration level of $\sim 4 \mathrm{mg} / \mathrm{l}$. At levels ranging from 3 to $5 \mathrm{mg} / \mathrm{l}$, water may appear opalescent and when boiled can form an oily film 
Table 5 Classification of groundwater based on EC, TDS, $\mathrm{TH}$, chloride, and nitrate

\begin{tabular}{|c|c|c|c|c|c|}
\hline & \multicolumn{2}{|c|}{ Gwandu formation } & \multicolumn{3}{|c|}{ Illo formation } \\
\hline & No. of samples & $\%$ of samples & $\begin{array}{l}\text { No. of } \\
\text { samples }\end{array}$ & $\%$ of samples & Classification \\
\hline \multicolumn{6}{|l|}{ (a) TDS (mg/l) } \\
\hline$<500$ & 7 & 35 & 17 & 85 & Essential for drinking \\
\hline $500-1000$ & 2 & 10 & 2 & 10 & Required for drinking \\
\hline $1000-3000$ & 10 & 50 & 1 & 5 & Suitable for drinking \\
\hline$>3000$ & 1 & 5 & 0 & 0 & $\begin{array}{l}\text { Not suitable for } \\
\text { irrigation and } \\
\text { drinking }\end{array}$ \\
\hline Total & 20 & 100 & 20 & 100 & \\
\hline \multicolumn{6}{|l|}{ (b) $E C$} \\
\hline Less than 250 & 6 & 30 & 17 & 85 & Excellent \\
\hline $250-750$ & 1 & 5 & 2 & 10 & Good \\
\hline 750-2000 & 2 & 10 & 1 & 5 & Permissible \\
\hline $2000-3000$ & 9 & 45 & - & - & Doubtful \\
\hline Above 3000 & 2 & 10 & - & - & Unsuitable \\
\hline Total & 20 & 100 & 20 & 100 & \\
\hline \multicolumn{6}{|l|}{ (c) Hardness } \\
\hline $0-75$ & 13 & 65 & 14 & 70 & Soft \\
\hline $75-150$ & 6 & 30 & 2 & 10 & Moderate Hard \\
\hline $150-300$ & 1 & 5 & 4 & 20 & Hard \\
\hline$>300$ & 0 & 0 & 0 & 0 & Very Hard \\
\hline Total & 20 & 100 & 20 & 100 & \\
\hline \multicolumn{6}{|l|}{ (d) Chloride } \\
\hline Less than 0.14 & - & - & - & - & Awfully fresh \\
\hline $0.14-0.85$ & - & - & - & - & Very fresh \\
\hline $0.85-4.23$ & 20 & 100 & - & - & Fresh \\
\hline $4.23-8.46$ & - & - & - & - & Fresh brackish \\
\hline $8.46-28.21$ & - & - & - & - & Brackish \\
\hline $28.21-546.13$ & - & - & 15 & 75 & Brackish salt \\
\hline Above 564.13 & - & - & 5 & 25 & Hyper saline \\
\hline Total & 20 & 100 & 20 & 100 & \\
\hline \multicolumn{6}{|l|}{ (e) Nitrate } \\
\hline Less than 5 & 7 & - & 1 & 5 & Acceptable \\
\hline $5-30$ & 13 & - & 19 & 95 & Moderate \\
\hline Above 30 & & - & - & - & Severe \\
\hline Total & 20 & 100 & 20 & 100 & \\
\hline
\end{tabular}

[40]. Natural aquifers hardly contain $\mathrm{Zn}^{2+}$ above $1 \mathrm{mg} / \mathrm{l}$. However, $\mathrm{Zn}^{2+}$ concentration can be elevated by the effects of galvanized pipes, and this can inform cadmium elevation from older pipes. Overall, the concentration of cations has been greater in the Illo aquifer. Chloride and $\mathrm{SO}_{4}{ }^{2-}$ were outstanding anions in Illo and Gwandu aquifers respectively (Table 4, Fig. 5). While excessive $\mathrm{Cl}^{-}$consumption is not related to serious health risks, at a level above $250 \mathrm{mg} / \mathrm{l}$, a salty taste is noticed. Variations in $\mathrm{Cl}^{-}$level ( $\sim 5 \mathrm{mg} / \mathrm{l})$, at a sampling site compared to another sampling point, may suggest contamination of groundwater from sewage, particularly if the $\mathrm{NH}_{4}$ level is also raised.
Sulfate is mainly derived from rock minerals; excessive ingestion in drinking water can be connected with emetic effects especially if considered in conjunction with $\mathrm{Mg}^{2+}$ and/or $\mathrm{Na}^{+}$. Carbonate and bicarbonate concentrations differ significantly. When $\mathrm{CO}_{3}{ }^{2-}$ and $\mathrm{HCO}_{3}{ }^{-}$are joint with $\mathrm{Ca}^{2+}$ and $\mathrm{Mg}^{2+}$, they form carbonate hardness [69]. Similarly, if soil distillates under dry conditions, it advances as $\mathrm{CaCO}_{3}$ or $\mathrm{MgCO}_{3}$. Consequently, $\mathrm{Ca}^{2+}$ and $\mathrm{Mg}^{2+}$ concentrations decrease relative to $\mathrm{Na}^{+}$concentration and the sodium adsorption ratio (SAR) rises $[107,108]$. This causes an alkalizing effect and elevated pH levels in aquifers. When groundwater analysis shows a high $\mathrm{pH}$ level, it 

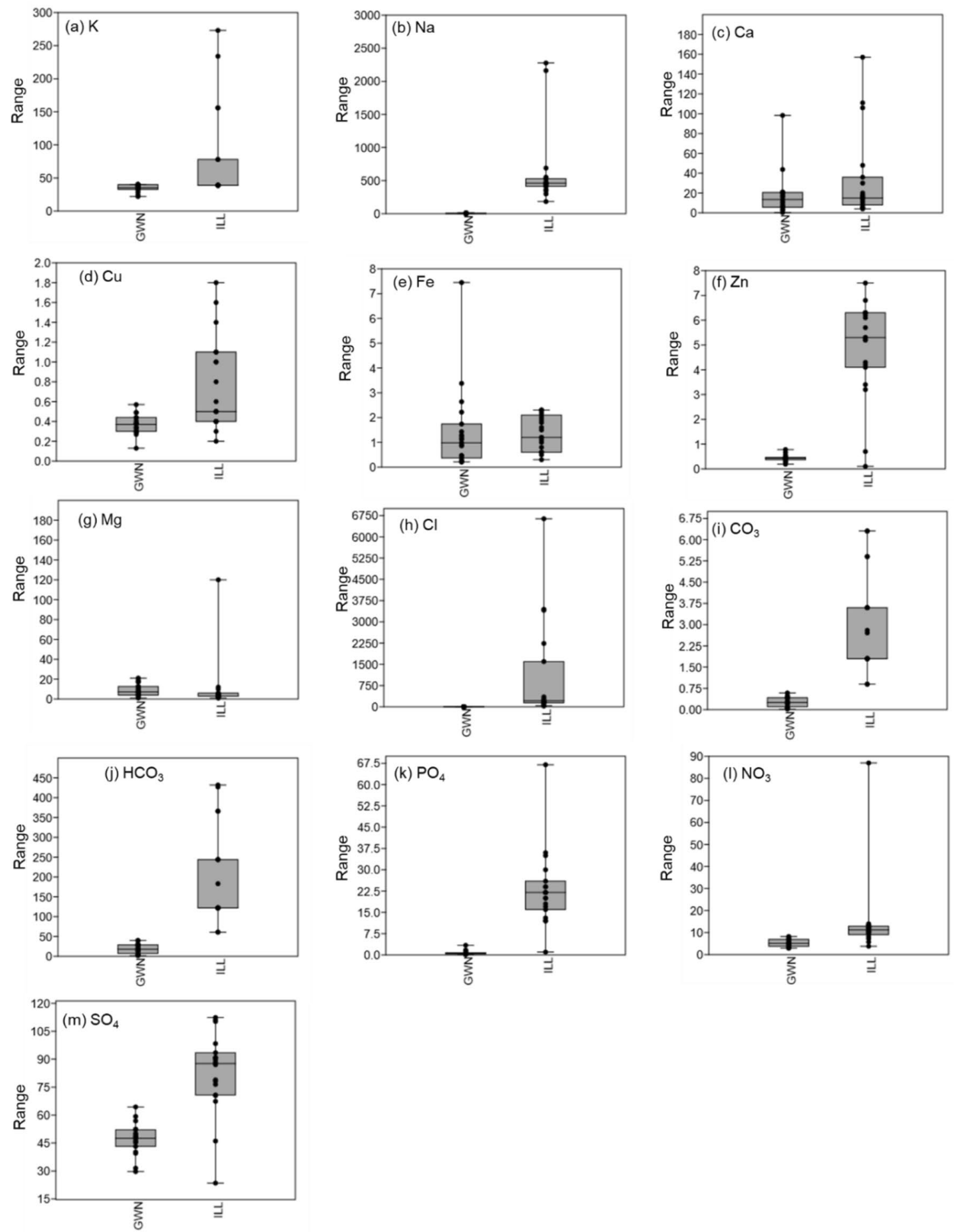

Fig. 5 Variability of chemical parameters; a potassium; b sodium; c calcium; $\mathbf{d}$ copper; $\mathbf{e}$ iron; $\mathbf{f}$ Zinc; $\mathbf{h}$ magnesium; $\mathbf{h}$ chloride; i carbonate; $\mathbf{j}$ bicarbonate; $\mathbf{k}$ phosphate; I nitrate; and $\mathbf{m}$ sulfate. Note: The entire ionic concentrations are in $\mathrm{mg} / \mathrm{l}$

indicates a high amount of carbonate/bicarbonate ions [44].

Nitrate and $\mathrm{PO}_{4}{ }^{3-}$ concentrations differ significantly. High $\mathrm{NO}_{3}{ }^{-}$concentration in groundwater can be linked to past anthropogenic activities, such as manure slurries, other organic wastes, and artificial fertilizers. Nitrate is primarily derived from the application of fertilizers and oxidation of $\mathrm{NH}_{4}$. Also, the origin of nitrate can originate 
from the nitrogen of soil, the $\mathrm{NO}_{3}{ }^{-}$of precipitation and fuel combustion $[63,68,112]$. Similarly, the presence of $\mathrm{PO}_{4}{ }^{3-}$ in groundwater aquifers can also be linked to anthropogenic activities, since $\mathrm{PO}_{4}{ }^{3-}$ is increasingly derived from municipal sewage and household chemicals such as detergent. The significance of $\mathrm{PO}_{4}{ }^{3-}$ is mostly linked to eutrophication of surface water bodies. $\mathrm{High}_{4}{ }^{3-}$ and $\mathrm{NO}_{3}{ }^{-}$in water help plant and algal growths which can lead to a variation of diurnal dissolved oxygen, blooms, and littoral slimes [51, 99]. Groundwater is "Fresh" in Gwandu aquifer based on chloride concentration. However, groundwater from the Illo aquifer falls into the "Brackish Salt-Hyper Saline" class. No severe nitrate pollution was noticed in the study area (Table 5), except in the sampling location (Sp17) under Illo formation.

\subsection{Groundwater evolution}

\subsubsection{Rock weathering and ion exchange process}

The relationship between dissolved elements in aquifers indicates the source of solutes and the processes that produced the detected groundwater properties. It is assumed that the dissolution of carbonate produces substantial parts of $\mathrm{HCO}_{3}{ }^{-}$, through the action of recharge waters enriched in $\mathrm{CO}_{2}$ derived from the atmosphere $[108,109]$. During infiltrating through the unsaturated zone, the rainwater with little dissolved $\mathrm{CO}_{2}$ from air mainly dissolve $\mathrm{CO}_{2}$ from soil which initially derived from air. Thus, the recharging water enriched in $\mathrm{CO}_{2}$ directly deriving from soil [28, 67]. Consequently, a $\mathrm{Ca}-\mathrm{HCO}_{3}$ water type is formed once $\mathrm{Ca}^{2+}$ is freed by the disbanding of carbonate ions. Correlations between $\mathrm{Ca}^{2+}, \mathrm{HCO}_{3}{ }^{-}, \mathrm{Mg}^{2+}$, and $\mathrm{Na}^{+}$provide vibrant evidence relating to the process of the stoichiometry. Table 6 presents a correlation matrix between hydrochemical elements. There is a positive but weak correlation between $\mathrm{Ca}^{2+}$ and $\mathrm{HCO}_{3}{ }^{-}$in Gwandu aquifer $(r=0.25)$, suggesting $\mathrm{Ca}^{2+}$ was not derived from calcite mineral; in Illo aquifer, the two ions correlate significantly $(r=0.53)$. However, a very weak positive correlation between $\mathrm{Ca}^{2+}$ and $\mathrm{SO}_{4}{ }^{2-}$ indicate that groundwater samples are far from 1:1 line, further suggesting that $\mathrm{Ca}^{2+}$ was not derived from gypsum [19]. Calcium is derived naturally from many solid rocks, mainly limestone, dolomite, and gypsum [108, 109]. A charge balance is expected to occur between cations and anions when $\mathrm{HCO}_{3}{ }^{-}, \mathrm{SO}_{4}{ }^{2-}, \mathrm{Ca}^{2+}$, and $\mathrm{Mg}^{2+}$ are derived from calcite. Groundwater in the Sokoto basin is deficient of $\left[\mathrm{Ca}^{2+}+\mathrm{Mg}^{2+}\right]$ relative to $\left[\mathrm{HCO}_{3}{ }^{-}+\mathrm{SO}_{4}{ }^{2-}\right]$, and $\left[\mathrm{HCO}_{3}{ }^{-}+\mathrm{SO}_{4}{ }^{2-}\right]$ relative to $\left[\mathrm{Ca}^{2+}+\mathrm{Mg}^{2+}\right]$ suggesting possible leaching of these ions from landfills, municipal sewage, and industrial effluents. Thus, excess positive charge between $\mathrm{Ca}^{2+}$ and $\mathrm{Mg}^{2+}$ must be balanced by $\mathrm{Cl}^{-}$, the only anion. Significant correlations between TDS and
$\mathrm{Mg}^{2+}(r=0.70), \mathrm{TDS}$, and $\mathrm{NO}_{3}{ }^{-}(r=0.57)$ in Gwandu aquifer, suggest that TDS in Gwandu aquifer is derived from dissolution of $\mathrm{Mg}^{2+}$ and $\mathrm{NO}_{3}{ }^{-}$ions.

Likewise, TDS correlates significantly with $\mathrm{Ca}^{2+}$ in the Illo aquifer and correlates weakly but positively with $\mathrm{CO}_{3}{ }^{2-}(r=0.39), \mathrm{HCO}_{3}{ }^{-}(r=0.40)$, and $\mathrm{NO}_{3}{ }^{-}(r=0.34)$, suggesting TDS was derived from dissolution of $\mathrm{Ca}^{2+}, \mathrm{CO}_{3}{ }^{2-}$, and $\mathrm{SO}_{4}{ }^{2-}$ ions. A significant correlation between TDS and $\mathrm{Ca}^{2+}$ explained the hard water (20\%) in Illo and moderately hard water (30\%) in Gwandu aquifers. The TH correlates significantly with $\mathrm{Ca}^{2+}$ in Gwandu ( $\left.r=0.91\right)$ and weakly correlated with $\mathrm{Mg}^{2+}(r=0.41)$ and $\mathrm{Fe}^{3+}(r=0.34)$. Hardness is controlled by many factors such as a geochemical evolution of groundwater, outflow from adjacent formations, and sediment/rock composition. Possible anthropogenic control on hardness includes saltwater intrusion induced by pumping and irrigation return flows [52].

\subsubsection{Sodium-chloride ratio}

The mechanism for obtaining salinity in arid and semiarid environments can be identified using the relationship between $\mathrm{Na}^{+}$and $\mathrm{Cl}^{-}$to evaluate the process of silicate weathering [75]. A uniform concentration of $\mathrm{Na}^{+}$and $\mathrm{Cl}^{-}$in groundwater aquifer is obtained through the dissolution of halite. The positive correlation between the two ions suggests that $\mathrm{Na}^{+}$is derived from halite. Basic $\mathrm{Na}^{+}$is correlated to class 2 water hazard, i.e., elevated $\mathrm{Na}^{+}$concentrations in potable water are linked to health hazards. Nevertheless, $\mathrm{NaCl}$ poses slight or no danger and is linked to a Class 1 water hazard. The implication of regulating $\mathrm{Na}^{+}$ in water is its joint impact with $\mathrm{SO}_{4}{ }^{2-}$. Usually, $\mathrm{Na}^{+}$levels in aquifers depend on the attending anion [100]. Accordingly, $60 \%$ of water samples derived from Gwandu aquifer have molar ratios greater than 1 . Correspondingly, $75 \%$ of groundwater samples derived from Illo Formation have a molar ratio greater than 1 , suggesting silicate weathering as the basis for $\mathrm{Na}^{+}$. It is important to understand that cation exchange between $\mathrm{Na}^{+}$and $\mathrm{Ca}^{2+}$ or $\mathrm{Mg}^{2+}$ may cause high levels of $\mathrm{Na}^{+}$in aquifers. If an aquifer has a molar ratio above 1 , it signposts absence of $\mathrm{Mg}^{2+}+\mathrm{Ca}^{2+}$ which is equivalent to $\mathrm{Ca}^{2+}-\mathrm{Na}^{+}$cation exchange process. Thus, soft water can be formed. Aquifers having clay mineral and $\mathrm{Na}^{+}$ resulting from the exchangeable sites can exchange with $\mathrm{Ca}^{2+}$ and $\mathrm{Mg}^{2+}$ and initiates higher $\mathrm{Na}^{+}$levels.

The ion exchange process between rock mineral and groundwater during recharge or transit and residence time was further evaluated using the Schoeller index (Si) [94]. The Si values tend to be positive if $\mathrm{Na}^{+}$and $\mathrm{K}^{+}$ exchange with $\mathrm{Ca}^{2+}$ and $\mathrm{Mg}^{2+}$, suggesting chloro-alkaline equilibrium. The computed values of Schoeller indices are presented in Table 2. Chloro-alkaline disequilibrium is indicated by the negative Schoeller index. The indices 


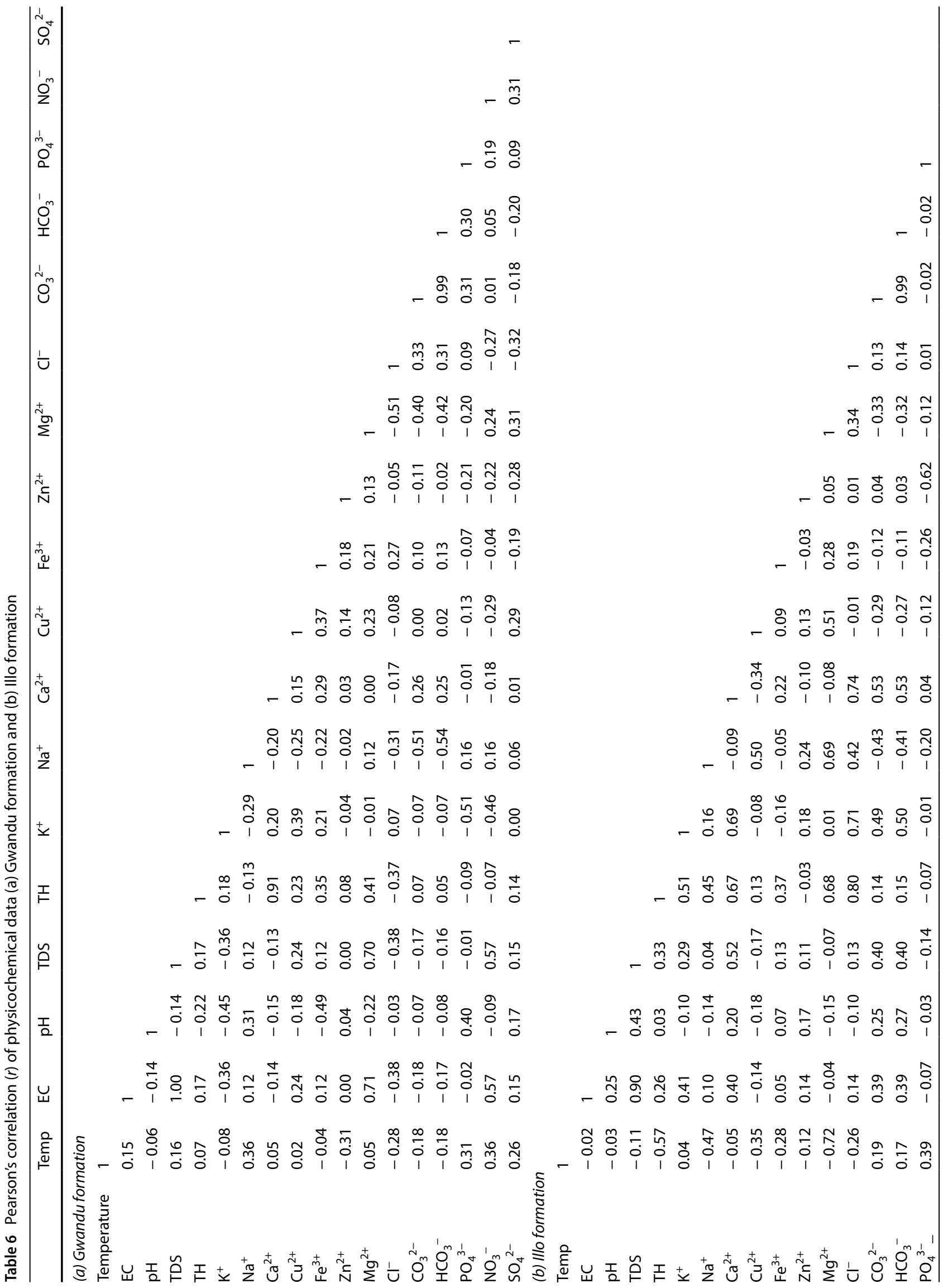




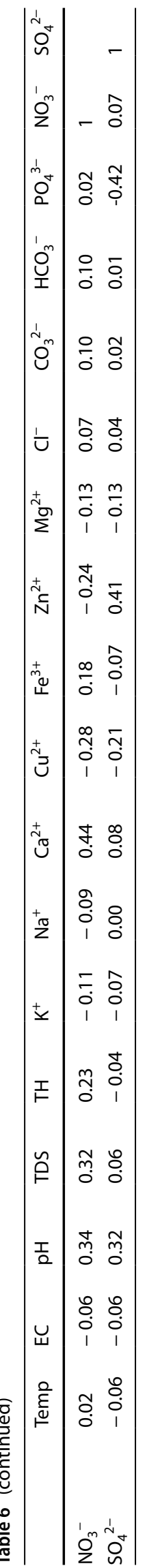

were positive in both Gwandu and Illo aquifers, indicating overall base-exchange reaction. In groundwater aquifers having chloro-alkaline equilibrium, the alkaline earths are exchanged with $\mathrm{Na}^{+}$ions $\left(\mathrm{HCO}_{3}{ }^{-}>\mathrm{Mg}^{2+}+\mathrm{Ca}^{2}\right)$. These types of aquifers are classified as base exchange soft water. However, aquifers in which $\mathrm{Na}^{+}$ions are exchanged with alkaline earths $\left(\mathrm{Mg}^{2+}+\mathrm{Ca}^{2+}>\mathrm{HCO}_{3}{ }^{-}\right)$are classified as hardened water $[108,109]$.

\subsubsection{Hydrogeochemical facies}

The evolution of groundwater is largely dependent on the geological properties of the aquifer rock minerals in the absence of anthropogenic inputs [109]. Piper trilinear diagram is used to classify groundwater base on the basic geochemical properties of the ionic concentrations. Results showed that the alkaline earth $(\mathrm{Ca}+\mathrm{Mg})$ exceeds alkalis $(\mathrm{Na}+\mathrm{K})$ in three sampling sites over Gwandu formation; the alkalis exceed alkaline earth in 17 sampling locations (Table 7). The strong acids exceed weak acids in all the 20 sampling locations. However, the alkalis exceed alkaline earth in the Illo aquifer; the strong acids exceed weak acids in 16 sampling locations.

The Piper [87] trilinear diagram (Fig. 6) revealed a $\mathrm{Ca}-\mathrm{Mg}-\mathrm{HCO}_{3}$ water type with mixed $\mathrm{Na}-\mathrm{Mg}-\mathrm{Cl}$ water type in Gwandu aquifer; $\mathrm{Na}-\mathrm{Cl}-\mathrm{HCO}_{3}$ and mixed $\mathrm{Cl}-\mathrm{CO}_{3}-\mathrm{HCO}_{3}$ in the Illo aquifer. Groundwater in the Sokoto basin is chiefly of two facies: $\mathrm{Ca}-\mathrm{Mg}-\mathrm{HCO}_{3}$ and $\mathrm{Ca}-\mathrm{Mg}-\mathrm{SO}_{4}-\mathrm{Cl}$. The facies conceivably are resultant of the dissolution of $\mathrm{Ca}^{2+}$ and $\mathrm{Mg}^{2+}$ carbonates ions [12].

\subsubsection{Gibbs diagram}

The mechanism controlling groundwater chemistry was also assessed using Gibbs's plot. The model is a plot of weight ratio of TDS versus $[\mathrm{Cl}] /\left[\mathrm{Cl}+\mathrm{HCO}_{3}\right]$ and $[\mathrm{Na}+\mathrm{K}] /$ $\mathrm{Na}+\mathrm{K}+\mathrm{Ca}$ ] for anions and cations (Fig. 7). Rock weathering is the major mechanism controlling the hydrochemistry of aquifers in both Gwandu and Illo aquifers. Current findings concur with Gibbs [45]. Water bodies in semiarid regions of the world drive their salts from soils and rocks of their basins. Their outlook in this group is dependent on climate and topography and the configuration of the rock material in their respective basins $[45,73]$. The lithology of the study area is mainly comprised of sands with interbedding of clay layers of diverse textural classes superimposing a Crystalline Basement Complex formation [59, 83].

\subsubsection{Saturation index of rock minerals}

The supersaturation of primary minerals or secondary mineral precipitation is indicated by positive SI values. In contrast, negative SI values indicate the dissolution 
Table 7 Groundwater characterization following Piper [87] trilinear diagram

\begin{tabular}{|c|c|c|c|c|}
\hline $\begin{array}{l}\text { Geochemical } \\
\text { facies zone }\end{array}$ & Groundwater quality characterization & Samples ID & $\begin{array}{l}\text { No. of sam- } \\
\text { ples }\end{array}$ & $\%$ of samples \\
\hline \multicolumn{5}{|c|}{ (a) Gwandu formation } \\
\hline 1 & Alkaline earth $(\mathrm{Ca}+\mathrm{Mg})$ exceed alkalis $(\mathrm{Na}+\mathrm{K})$ & Sp4, Sp9, Sp15 & 3 & 15 \\
\hline 2 & Alkalis exceed alkaline earth & $\begin{array}{l}\text { Sp1-Sp3, Sp5-Sp8, Sp10- } \\
\text { Sp14, Sp16-Sp20 }\end{array}$ & 17 & 75 \\
\hline 3 & Weak acids $\left(\mathrm{HCO}_{3}+\mathrm{CO}_{3}\right)$ exceeds strong acid $\left(\mathrm{SO}_{4}+\mathrm{Cl}\right)$ & -0 & 0 & 0 \\
\hline 4 & Strong acids exceed weak acids & Sp1-Sp20 & 20 & 100 \\
\hline \multicolumn{5}{|c|}{ (b) Illo formation } \\
\hline 1 & Alkaline earths $(\mathrm{Ca}+\mathrm{Mg})$ exceed alkalis $(\mathrm{Na}+\mathrm{K})$ & 0 & 0 & 0 \\
\hline 2 & Alkalis exceed alkaline earth & Sp1-Sp20 & 20 & 100 \\
\hline 3 & Weak acids $\left(\mathrm{HCO}_{3}+\mathrm{CO}_{3}\right)$ exceeds strong acid $\left(\mathrm{SO}_{4}+\mathrm{Cl}\right)$ & Sp1, Sp2, Sp7, Sp10 & 4 & 20 \\
\hline 4 & Strong acids exceed weak acids & Sp3-6, Sp8-9, Sp11-20 & 16 & 80 \\
\hline
\end{tabular}

Fig. 6 Piper trilinear diagram showing hydrogeochemical facies of groundwater

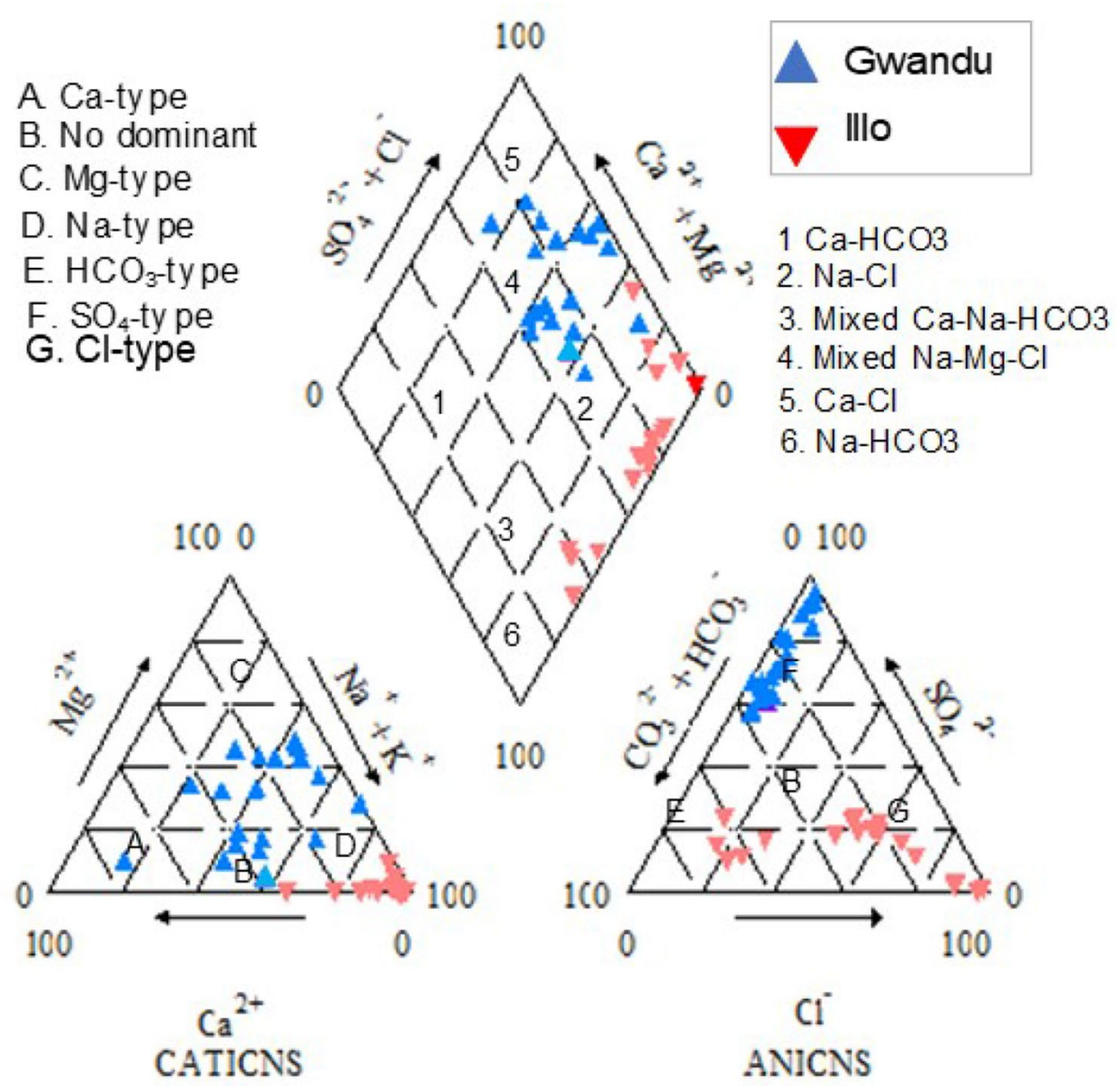

of minerals or undersaturation. An equilibrium condition is indicated by $\mathrm{SI}$ of $\pm 0.5[2,27]$. Table 3 summarized saturation indices (SIs) derived from geochemical modeling using PHREEQC (version 3.5.0.14000). The SI values for minerals in the study area are characterized by significant differences in chrysotile, goethite, gypsum, $\mathrm{H}_{2}(\mathrm{~g}), \mathrm{H}_{2} \mathrm{O}(\mathrm{g}), \mathrm{H}_{2} \mathrm{~S}(\mathrm{~g})$, illite, and sepiolite.

4.3.5.1 Carbonate minerals Precipitation of $\mathrm{CaCO}_{3}$ is derived from variation in $\mathrm{pH}$ since carbonate mineral 

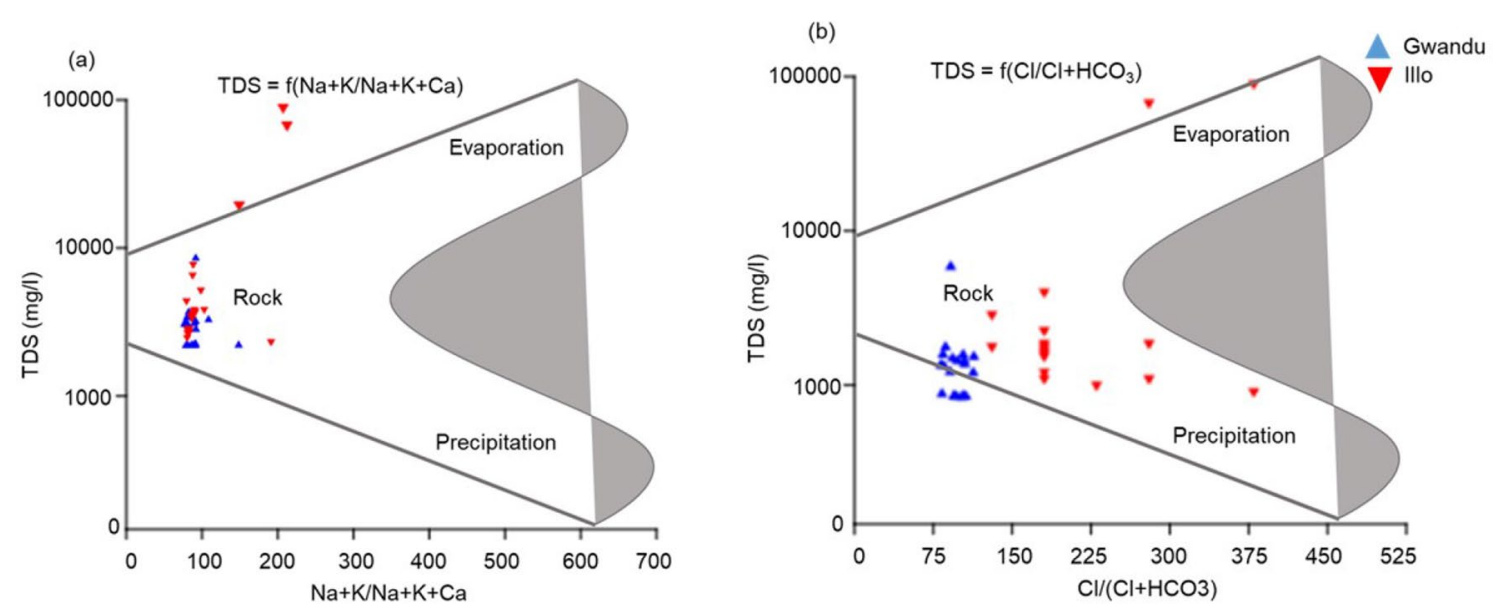

Fig. 7 Gibbs plot showing major natural mechanisms controlling groundwater chemistry

dissolution occurs consequent of changes in $\mathrm{HCO}_{3}{ }^{-}$and $\mathrm{pH}$. Carbonic acid is taken up and in a closed system not replaced as the dissolution continues in the outside system. The Sls for Gwandu and Illo aquifers for the carbonate minerals showed values for dolomite and calcite are in a state of equilibrium. However, the SI values for aragonite are undersaturated (Fig. 8a). Significant differences in the Sls values for the carbonate minerals aid in identifying the potential discharge/recharge zones $[2,27]$.

4.3.5.2 Silica Dissolved silica can reach amorphous $\left[\mathrm{SiO}_{2}(\mathrm{a})\right]$, cryptocrystalline (Chalcedony: $\mathrm{CHAL}$ ), or crystalline (quartz: QTZ) form. The SI values for Gwandu and Illo aquifers (Fig. 8b) showed that the amorphous form is undersaturated with slight saturation of crystalline form. The SI values for cryptocrystalline form and mineral quartz $\left(\mathrm{SI}_{\mathrm{QTZ}}\right)$ show undersaturation. The $\mathrm{SI}$ values for the silica mineral are in the following order: $\mathrm{SI}_{\mathrm{Si}_{2}(\mathrm{a})}>\mathrm{SI}_{\mathrm{CHAL}}>\mathrm{SI}_{\mathrm{QTZ}}$ (crystalline $>$ cryptocrystalline $>$ amorphous) in the Gwandu aquifer; $\mathrm{SI}_{\mathrm{SiO}_{2}(\mathrm{a})}>\mathrm{SI}_{\mathrm{QTZ}}>\mathrm{SI}_{\mathrm{CHAL}}$ (amorphous $>$ crystalline $>$ cryptocrystalline $>$ ) in the Illo aquifer. At the higher limit of dissolved silica content of natural groundwater aquifers, a metastable phase can be found for most low-temperature processes $[19,27]$. The high solubility of silica at low temperatures is limited by amorphous silica instead of quartz.

4.3.5.3 Aluminosilicates minerals The consistent dissolution of amino-silicate minerals in aquifers is one of the primary weathering reactions. The secondary mineral is derived from the conversion of primary mineral. The release of cation and silicic acid is consequent of the structural breakdown of amino silicates. Consequently, alkalinity is divulged to the dissolved phase derived from the bases of the minerals. The Al is typically preserved at the time of reaction; the solid deposit tends to have an elevated Al content greater than the primary silicates. Amino-silicate mineral dissolution in aquifers is significantly affected by the chemically hostile nature of groundwater [27]. The SI values for secondary minerals (Fig. $8 \mathrm{c}$ are in the following order: K-mica $>\mathrm{K}$-felspar $>$ kaolinite $>$ Ca-montmorillonite $>$ gibbsite in the Gwandu aquifer; K-mica $>$ kaolinite $>$ K-Feldspar $>$ gibbsite $>$ Ca-montmorillonite in the Illo aquifer, respectively. The SI values for gibbsite are more variable than K-feldspar suggesting the discrepancy of rock weathering [27].

\subsection{Detection of potential discharge and recharge zones}

Sampling sites having positive SI values for gibbsite show the initial phases of rock weathering, indicative of recently recharged waters [27]. However, sites having positive SI values for K-feldspar show severe phases of rock weathering, which is typically reached by the prolonged residence time of the water in the aquifer. This can be used as an indicator of the discharge zones (Table 8). Sampling locations Sp2, Sp4, Sp8, Sp10, Sp11, and Sp13 as well as Sp7, Sp10, Sp11, Sp15, and Sp19 showed positive SI values for gibbsite, indicative of potential recharge zones over Gwandu and Illo formations. All the sampling locations in both Gwandu and Illo aquifers showed positive SI values for K-feldspar, indicative of groundwater discharge. Aquifers of Sokoto basins received recharge from the outcrop areas and discharges water at some locations particularly along with the floodplain areas of rivers draining the Sokoto basin or deep valleys.

\subsection{Multivariate analysis}

\subsubsection{Factor analysis}

The result of FA comprising of the loading scores, percentages, and eigenvalues of the total variance is 
Fig. 8 Saturation index of carbonate minerals, a aragonite, calcite, and dolomite, b saturation index of silicate minerals chalcedony, quartz and $\mathrm{SiO}_{2}$, and saturation index for aluminosilicates, c Ca-montmorillonite, gibbsite, K-feldspar, K-mica, and kaolinite

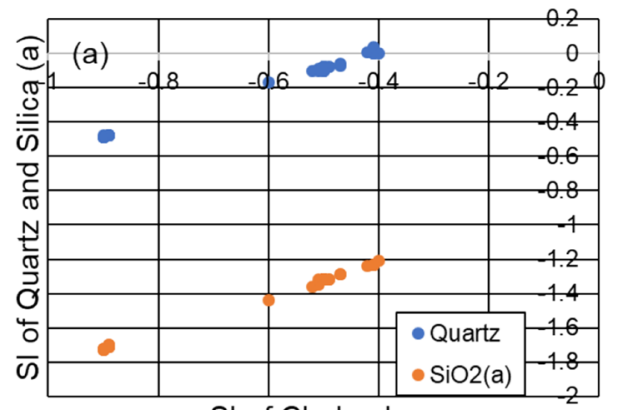

SI of Chalcedony
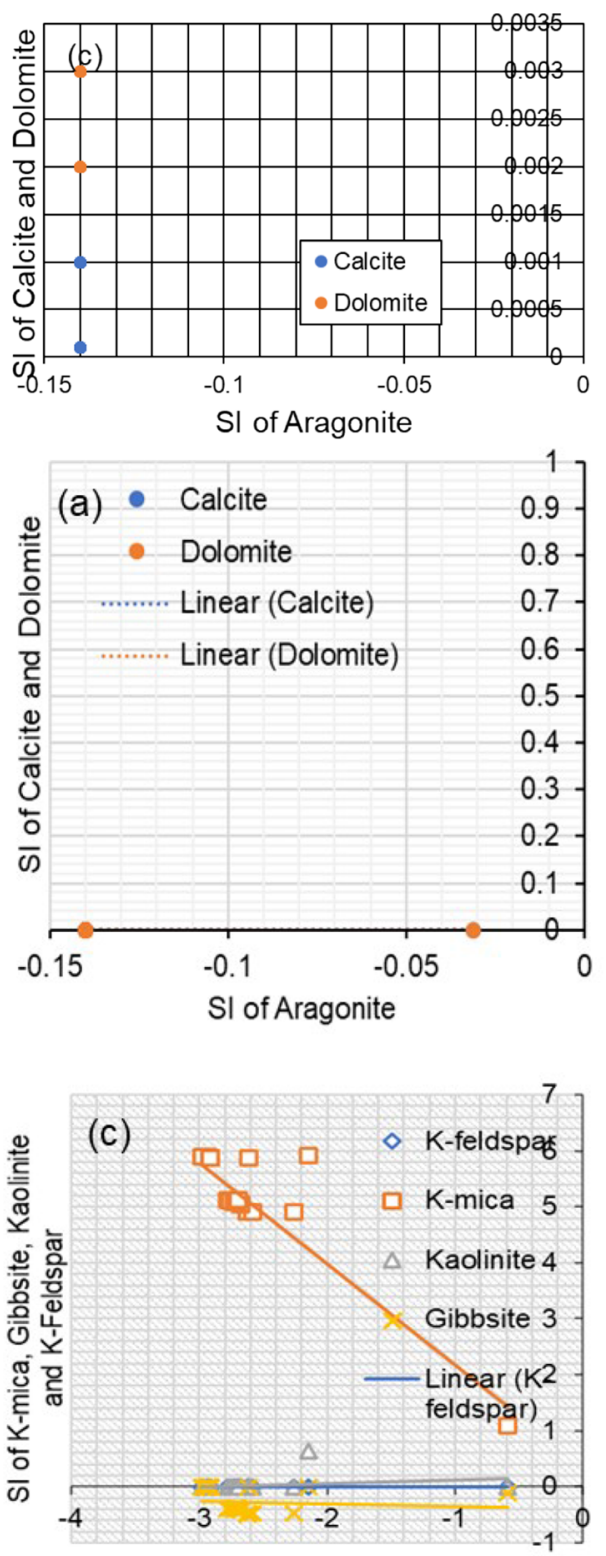

SI of Ca-Montrillionite

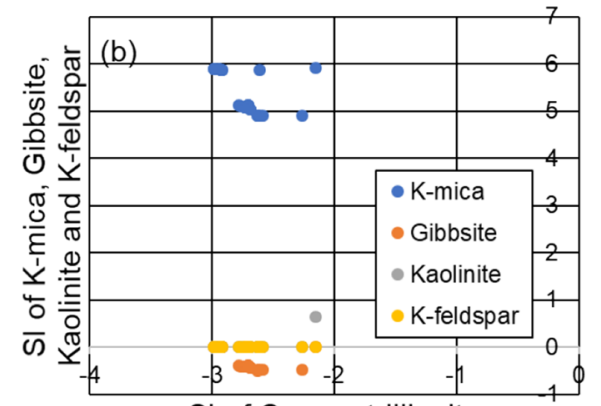

SI of Ca-montrillionite

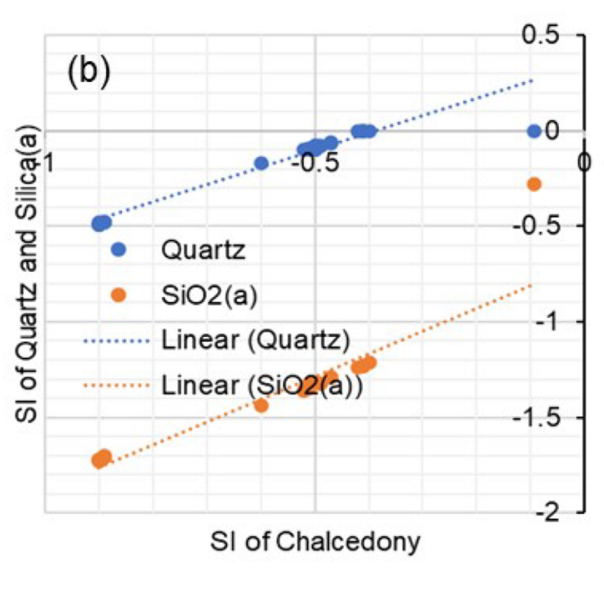

presented in Table 9. The abstraction of five Factors was based on the percentage of variance accumulated. It included a percentage above $70 \%[65,66]$. The five factors explained $71.62 \%$ and $77.62 \%$ of the total variance in Gwandu and Illo formations, respectively. Based on the scree test, the five factors having typical factor loadings implied five different noticeable contributions were involved in governing the hydrochemistry of aquifers in 
Table 8 Identification of recharge/discharge sites by saturation indices

\begin{tabular}{lllc}
\hline Geological formations & Saturation index & Fully saturated & $\begin{array}{l}\text { Potential } \\
\text { recharge/dis- } \\
\text { charge zones }\end{array}$ \\
\hline Gwandu formation & Gibbsite & Sp2, Sp4, sp8, Sp10, Sp11, Sp13 & 6 \\
& K-feldspar & Sp1-20 & 20 \\
Illo formation & Gibbsite & Sp7, Sp10, Sp11, Sp15, Sp19 & 5 \\
& K-feldspar & Sp1-20 & 20 \\
\hline
\end{tabular}

Table 9 Factor analysis scores (Varimax rotation) of physicochemical parameters

\begin{tabular}{|c|c|c|c|c|c|c|c|c|c|c|}
\hline \multirow[t]{3}{*}{ Parameter } & \multicolumn{5}{|c|}{ Gwandu formation } & \multicolumn{5}{|c|}{ Illo formation } \\
\hline & \multicolumn{5}{|c|}{ Components } & \multicolumn{5}{|c|}{ Components } \\
\hline & 1 & 2 & 3 & 4 & 5 & 1 & 2 & 3 & 4 & 5 \\
\hline Temp & 0.13 & -0.10 & 0.10 & -0.16 & 0.71 & -0.67 & -0.13 & -0.05 & -0.33 & -0.29 \\
\hline $\mathrm{EC}$ & 0.96 & 0.05 & 0.01 & -0.09 & 0.04 & 0.03 & 0.26 & 0.88 & -0.16 & 0.05 \\
\hline $\mathrm{pH}$ & -0.20 & -0.71 & -0.03 & -0.31 & -0.07 & -0.20 & -0.14 & 0.58 & 0.37 & 0.11 \\
\hline TDS & 0.96 & 0.04 & 0.01 & -0.08 & 0.04 & -0.02 & 0.21 & 0.93 & 0.16 & 0.04 \\
\hline TH & 0.15 & 0.19 & 0.94 & -0.01 & 0.01 & 0.51 & 0.74 & 0.15 & 0.36 & -0.04 \\
\hline $\mathrm{K}^{+}$ & -0.44 & 0.81 & 0.09 & -0.05 & 0.06 & -0.05 & 0.88 & 0.16 & -0.31 & 0.08 \\
\hline $\mathrm{Na}^{+}$ & 0.09 & -0.38 & -0.09 & -0.64 & 0.12 & 0.86 & 0.13 & 0.08 & -0.16 & 0.07 \\
\hline $\mathrm{Ca}^{2+}$ & -0.16 & 0.07 & 0.93 & 0.17 & 0.03 & -0.19 & 0.83 & 0.29 & 0.35 & -0.11 \\
\hline $\mathrm{Cu}^{2+}$ & 0.15 & 0.56 & 0.25 & 0.03 & 0.03 & 0.63 & -0.12 & -0.11 & -0.21 & 0.16 \\
\hline $\mathrm{Fe}^{3+}$ & 0.22 & 0.45 & 0.27 & 0.44 & -0.17 & 0.19 & 0.07 & -0.04 & 0.70 & 0.18 \\
\hline $\mathrm{Zn}^{2+}$ & 0.09 & -0.01 & 0.16 & -0.13 & -0.78 & 0.09 & -0.01 & 0.12 & -0.19 & 0.83 \\
\hline $\mathrm{Mg}^{2+}$ & 0.71 & 0.30 & 0.23 & -0.39 & -0.05 & 0.87 & 0.18 & -0.08 & 0.14 & 0.05 \\
\hline $\mathrm{Cl}^{-}$ & -0.36 & 0.04 & -0.39 & 0.60 & -0.17 & 0.30 & 0.87 & -0.07 & 0.12 & -0.07 \\
\hline $\mathrm{HCO}_{3}^{-}$ & -0.10 & -0.18 & 0.16 & 0.83 & -0.02 & -0.60 & 0.53 & 0.34 & -0.07 & 0.18 \\
\hline $\mathrm{CO}_{3}{ }^{2-}$ & 0.04 & -0.68 & 0.10 & 0.29 & 0.32 & -0.59 & 0.53 & 0.35 & -0.06 & 0.18 \\
\hline $\mathrm{PO}_{4}^{3-}$ & 0.67 & -0.26 & -0.15 & 0.10 & 0.43 & -0.13 & 0.02 & -0.03 & -0.20 & -0.88 \\
\hline $\mathrm{NO}_{3}^{-}$ & 0.13 & 0.06 & 0.15 & -0.31 & 0.60 & -0.19 & 0.05 & 0.19 & 0.72 & -0.24 \\
\hline Eigenvalues & 3.35 & 2.55 & 2.23 & 2.19 & 1.86 & 3.59 & 3.55 & 2.44 & 1.84 & 1.78 \\
\hline$\%$ of Variance & 19.70 & 14.97 & 13.12 & 12.90 & 10.93 & 21.10 & 20.87 & 14.33 & 10.85 & 10.48 \\
\hline Cumulative \% & 19.70 & 34.67 & 47.79 & 60.70 & 71.62 & 21.10 & 41.97 & 56.30 & 67.14 & 77.62 \\
\hline
\end{tabular}

All concentrations in $\mathrm{mg} / \mathrm{l}$, except Temp. $\left({ }^{\circ} \mathrm{C}\right), \mathrm{pH}$ (units) and EC $(\mu \mathrm{S} / \mathrm{cm}) @ 25^{\circ} \mathrm{C}$

Bold values indicate high positive loadings @ $\geq 0.65$ the study area. Factor 1 explained $19.70 \%$ of the total variance in Gwandu aquifer. It had high positive loadings on $\mathrm{EC}, \mathrm{Cl}^{-}, \mathrm{Mg}^{2+}$, and $\mathrm{PO}_{4}{ }^{2-}$, suggesting a strong geological and anthropogenic influence on groundwater. Substantial amounts of $\mathrm{PO}_{4}{ }^{2-}$ can be derived from household chemicals (e.g., detergents). Chloride is increasingly being added to the environment from sewage contamination and water softeners $[36,57,62,113]$. Similarly, in the Illo aquifer, Factor 1 explained $21.10 \%$ of the total variance. It had high positive loadings on $\mathrm{Na}^{+}$ and $\mathrm{Cl}^{-}$. Thus, it can be associated with both geological and anthropogenic effects. Factor 1 had eigenvalues of 3.35 and 3.59, respectively, indicating the greatest percentage relative to the remaining factors (Fig. 9).
Factor 2 explained $14.97 \%$ and $20.87 \%$ of the total variance in Gwandu and Illo aquifers, respectively. It had significant loading on $\mathrm{K}^{+}$in Gwandu aquifer and hardness, $\mathrm{K}^{+}$, and $\mathrm{CO}_{3}{ }^{2-}$ in the Illo aquifer. This factor can be linked to rock weathering. However, the observed negative loading on $\mathrm{pH}$ in this factor is deemed realistic since $\mathrm{pH}$ attained a converse relationship with ions of carbonate origin [65, 104]. The third factor explained $13.12 \%$ and $14.33 \%$ of the total variance. It had strong positive loadings on $\mathrm{Ca}^{2+}$ and TH in Gwandu aquifer and EC and TDS in Illo aquifer. Factor 4 had high positive loadings on $\mathrm{HCO}_{3}{ }^{2-}$ in Gwandu aquifer and $\mathrm{Zn}^{2+}$ and $\mathrm{SO}_{4}{ }^{2-}$ in Illo aquifer. These components are supposed to be divided by the two fractional contributions. The first $\left(\mathrm{Ca}^{2+}\right.$ and $\left.\mathrm{Zn}^{2+}\right)$ was connected to rock 

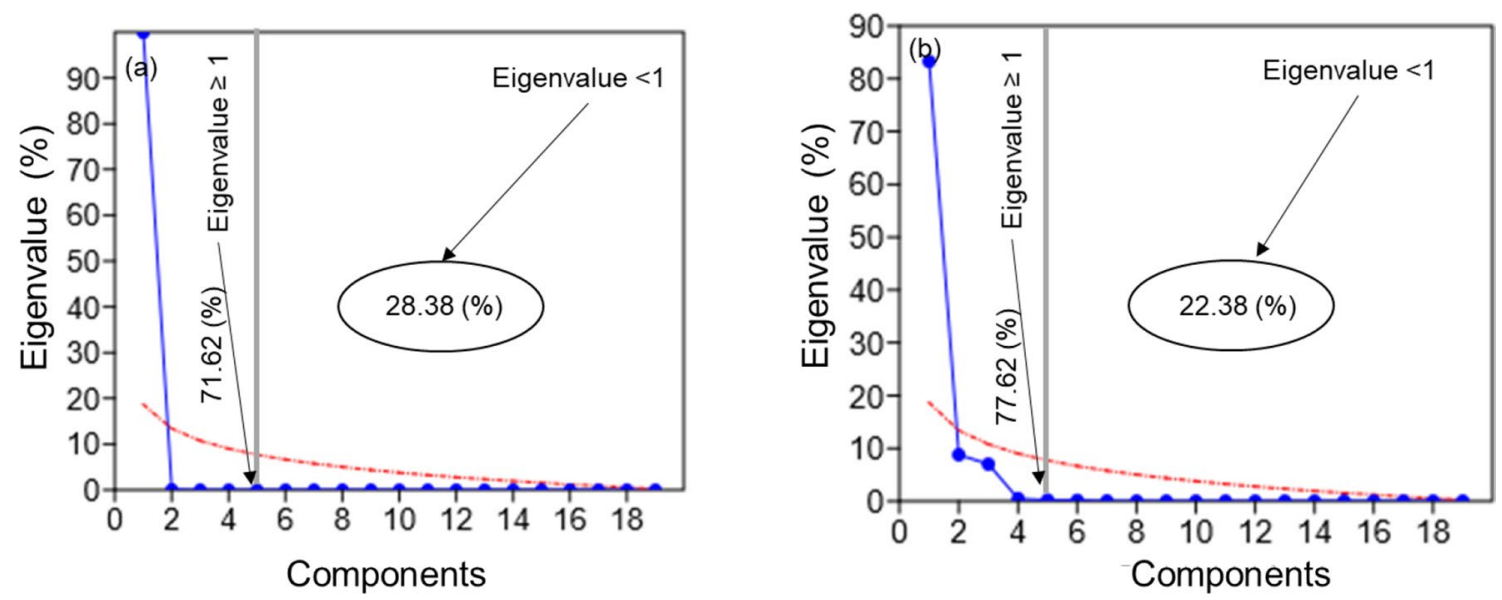

Fig. 9 Scree plot a Gwandu formation and b Illo formation

weathering. The second was connected to derivatives from anthropogenic sources, since $\mathrm{SO}_{4}{ }^{2+}$ can be derived from both geologic and anthropogenic sources.

Using a scatter plot, groundwater parameters showed a marked spatial variability and cluster well along with the bands of the two geologic environments. Factors 1 and 2 joined together explained $34.67 \%$ and $41.97 \%$ of the total variance in Gwandu and Illo aquifers, respectively (Fig. 10a). Despite the differential in geological settings, groundwater is primarily controlled by rock weathering. The results are notable since the parameters formed a relatively tight cluster around the geological environments. All the analyzed variables (or physicochemical parameters) are visible on the biplots, even though the parameters were chosen based on their absolute size(s) (i.e., high elemental concentrations). It is established by reference to the raw data matrix. So, the importance or otherwise of these elements can be recognized from this biplot. Though a good grouping is observed in the biplot of Factors 1 and 2, the biplot of Factors 2 and 3 (Fig. 10b) indicates a wide dispersity of groundwater parameters especially under Illo formation, making pattern identification difficult.

\subsubsection{Hierarchical cluster analysis}

The use of HCA in the hydrochemical analysis was confirmed to be logical by discerning hydrogeochemical data that behaves inversely. Using HCA, the sampling boreholes having similar hydrochemical properties were grouped into a separate cluster $[19,20,37]$. The graphics collections of the grouping process were offered as a dendrogram (Fig. 11a, b). Figure $11 \mathrm{a}$ is comprised of boreholes under Gwandu formation. The first cluster (or group) contained boreholes with analogous concentrations of elements such as temperature, $\mathrm{Na}^{+}, \mathrm{NO}_{3}{ }^{-}, \mathrm{SO}_{4}{ }^{2-}, \mathrm{pH}$, and $\mathrm{PO}_{4}{ }^{3-}$. Group 2 is comprised of the borehole with comparable concentrations of EC, TDS, and $\mathrm{Mg}^{2+}$.

Group 3 is comprised of wells with the similarity of $\mathrm{TH}_{\text {, }}$ $\mathrm{Ca}^{2+}, \mathrm{K}^{+}, \mathrm{Fe}^{3+}, \mathrm{Cl}^{-}, \mathrm{Zn}^{2+}, \mathrm{CO}_{3}{ }^{2-}, \mathrm{Cu}^{2+}$, and $\mathrm{HCO}_{3}{ }^{-}$. Likewise, in the Illo aquifer, boreholes with comparable concentrations of temperature, $\mathrm{pH}, \mathrm{EC}$, and TDS constituted Group 1. Group 2 is comprised of boreholes having similar concentrations of $\mathrm{TH}, \mathrm{Ca}^{2+}, \mathrm{K}^{+}, \mathrm{Cl}^{-}, \mathrm{Na}^{+} \mathrm{Cu}^{2+}, \mathrm{Mg}^{2+}$, and $\mathrm{Fe}^{3+}$. Group 3 is comprised of boreholes having similarities of $\mathrm{Zn}^{2+}, \mathrm{SO}_{4}{ }^{2-}$, $\mathrm{CO}_{3}{ }^{2-}, \mathrm{HCO}_{3}{ }^{-}, \mathrm{PO}_{4}{ }^{3-}$, and $\mathrm{NO}_{3}{ }^{-}$. This has further confirmed the impact of geological variability on the hydrochemical composition of groundwater. The clustering of hydrochemical parameters corresponded to geological variability.

\subsection{Suitability for irrigation use}

The USSL diagram (Fig. 12) showed that $40 \%$ of groundwater samples from Gwandu aquifer fall in low sodium-low-salinity class, $5 \%$ fall in low sodium-medium-salinity class, $35 \%$ fall in low-sodium high-salinity class, and $20 \%$ fall in low sodiumvery-high salinity class. Ninety percent ( $90 \%)$ of water samples from the Illo aquifer fall in low sodium-low-salinity class, $5 \%$ fall in low sodium-high-salinity class, and $5 \%$ fall in high sodium-high-salinity class. Groundwater in the study area can be used for irrigation with little or no risks of salinity hazard to crops. It has no risk of exchangeable $\mathrm{Na}^{+}$[105]. However, very low SAR and low salinity irrigation water (less than $200 \mathrm{\mu S} \mathrm{m}^{-1}$ ) disturbs the rates of water permeation into soils [48]. Thus, an evaluation of the permeability index may be required. 

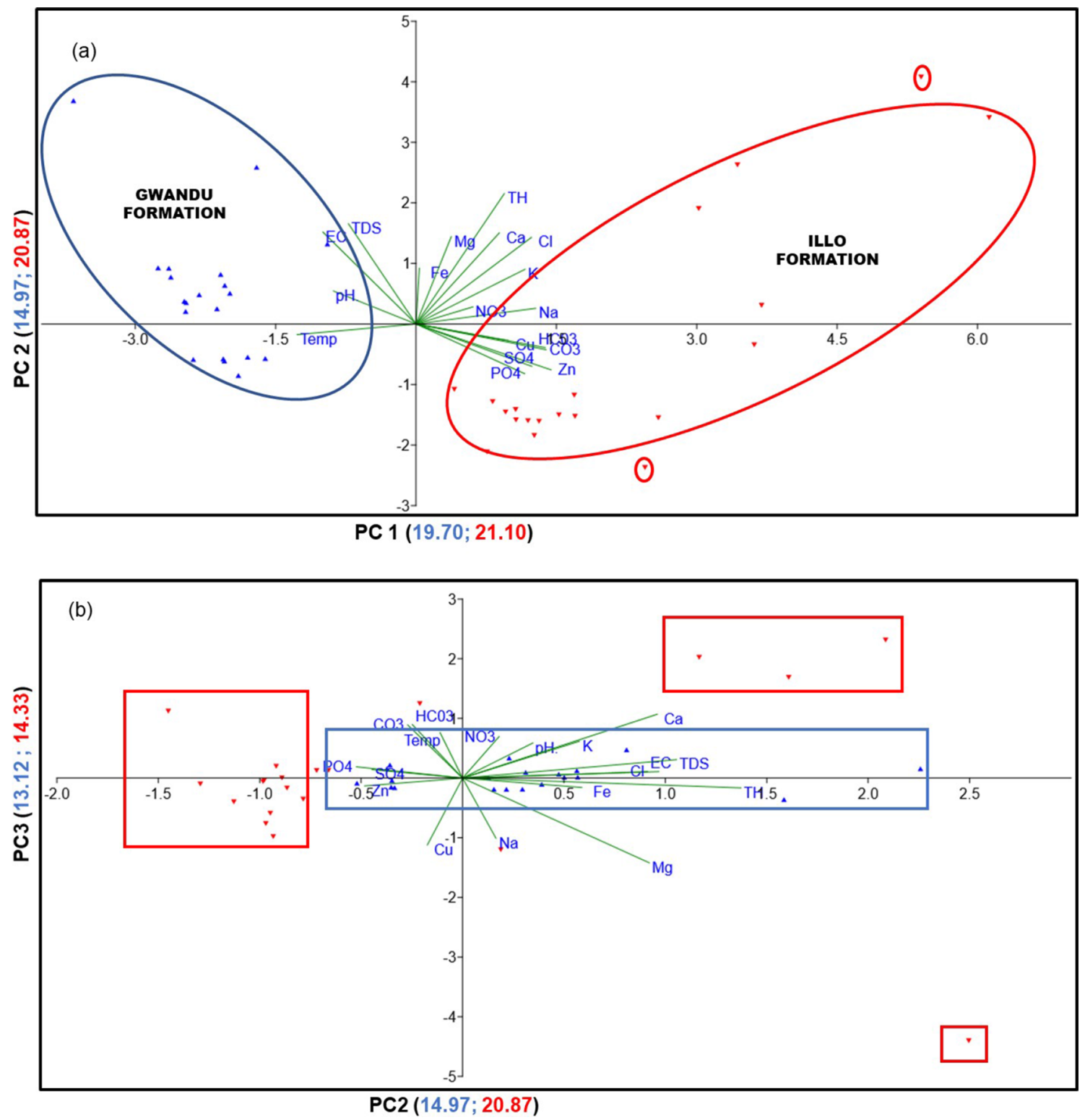

Fig. 10 Principal component analysis a biplot of PC 1 and $\mathbf{b} P C 2$ and $P C 2$ and PC3

\section{Conclusion}

The literature is concordant on the implication of understanding the hydrochemistry of groundwater aquifers. Evaluation of the hydrochemistry of Gwandu and IIlo aquifers showed a significant difference in physicochemical parameters of water quality and mineral facies of rock weathering. Results obtained from this study increased one's knowledge of how geological variability can influence the hydrochemistry of groundwater aquifers. The study found that the physicochemical parameters showed a significant difference between Gwandu and Illo formations. Groundwater classification based on physicochemical parameters revealed water of acceptable quality for drinking. The dominance of rock weathering is apparent as revealed by geochemical modeling and statistical analysis. Chrysotile, goethite, gypsum, $\mathrm{H}_{2}(\mathrm{~g}), \mathrm{H}_{2} \mathrm{O}(\mathrm{g}), \mathrm{H}_{2} \mathrm{~S}(\mathrm{~g})$, illite, and sepiolite minerals differ significantly between Gwandu and Illo formations. However, the SAR level was generally low. Thus, serious environmental problems might be expected with prolonged applications of these waters 
Fig. 11 The dendrogram was produced from cluster analysis based on the sampling wells to recognize the major hydrogeochemical physiognomies in the western Sokoto Basin (a) Gwandu

Dendrogram

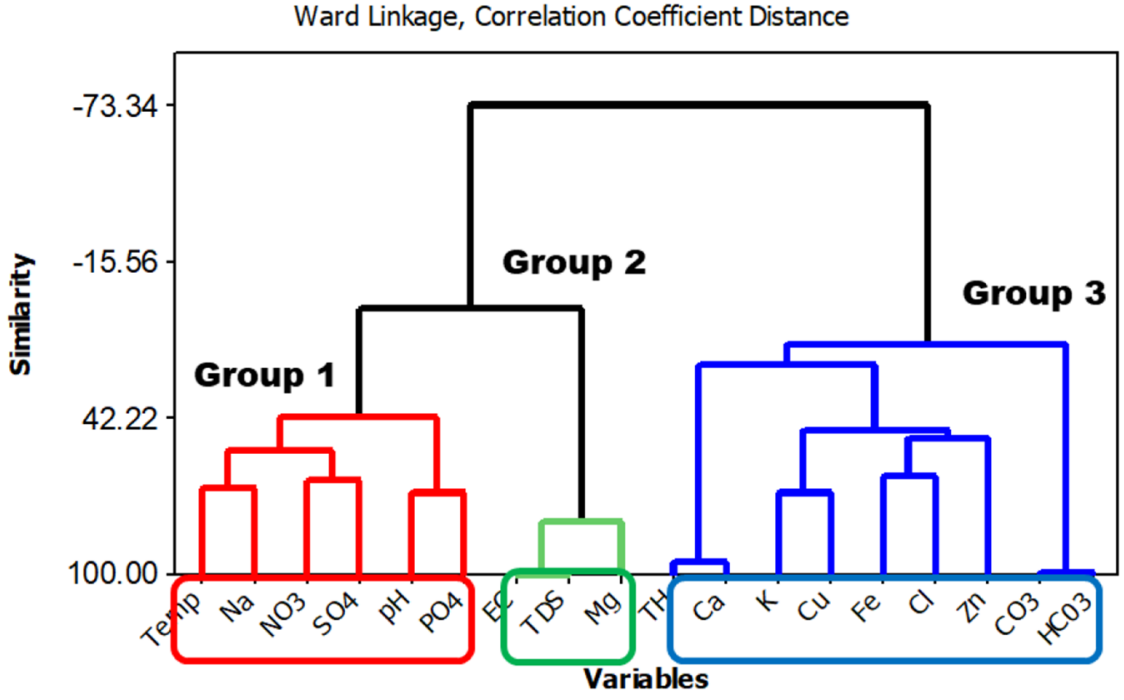

(b) Illo

Dendrogram

Ward Linkage, Correlation Coefficient Distance

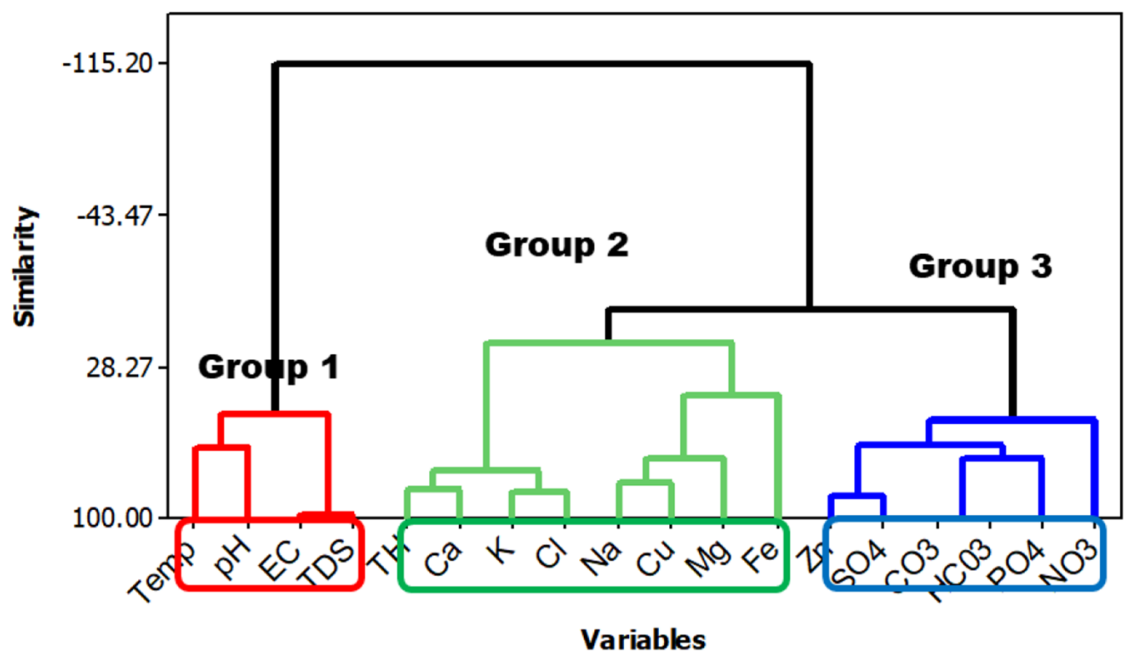

in irrigation fields. This study highlights the importance of understanding groundwater chemistry over different geological environments, using combined statistical techniques and geochemical modeling to show how geological variability can influence the hydrochemistry of aquifers. Hence, we hope that the findings from this study will stimulate others to an analogous method in a future study, especially those in arid and semi-arid environments. 
Fig. 12 Irrigation water classification based on USDA diagram

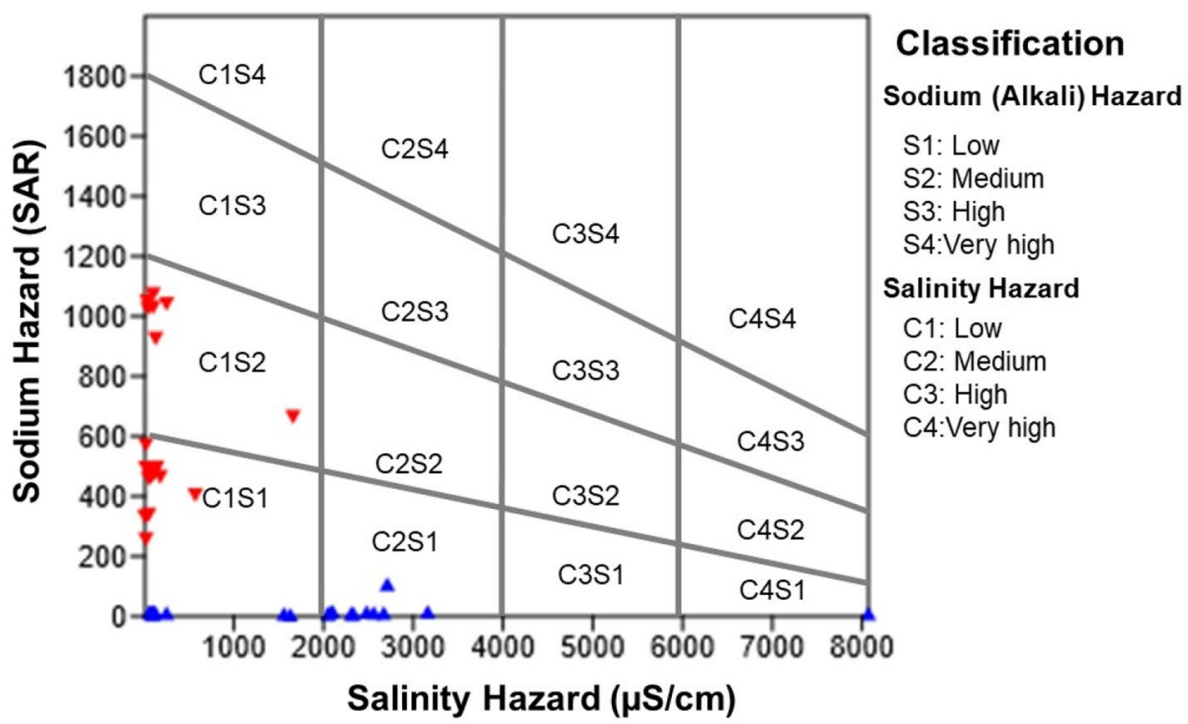

Acknowledgements This research was supported by Federal University Birnin kebbi. Thanks to all anonymous contributors. Our sincere thanks to anonymous contributors for their constructive comments which have helped improved the paper.

\section{Compliance with ethical standards}

Conflict of interest The authors declare that they have no conflict of interest.

\section{References}

1. Abdullahi SA, Muhammad MM, Adeogun BK, Mohammed UI (2014) Assessment of water availability in the Sokoto Rima River Basin. Resour Environ 4(5):220-233. https://doi. org/10.5923/j.re.20140405.03

2. Abo R, Merkel BJ (2015) Water quality of the Helvetian and Eocene aquifers in Al Zerba catchment and southern parts of Al Qweek Valley, Aleppo basin, Syria. Sustain Water Resour Manag 1(3):189-211. https://doi.org/10.1007/s4089 9-015-0019-2

3. Aboyeji OS, Eigbokhan SF (2016) Evaluations of groundwater contamination by leachates around Olusosun open dumpsite in Lagos metropolis, southwest Nigeria. J Environ Manag 183:333-341. https://doi.org/10.1016/j.jenvman.2016.09.002

4. Abusu CO (2019) Hydrogeochemical characterization of groundwater in Kankara, northwestern Nigeria. Sustain Water Resour Manag. https://doi.org/10.1007/s40899-019-00316-3

5. Adamu A (2019) Electrical resistivity mapping of aquiferous zones within Gudi-takalau area of Birnin Kebbi, Nigeria. ATBU J Environ Technol 12(1):29-46

6. Adelana SMA, Olasehinde PI, Vrbka P (2002) Groundwater recharge in the cretaceous and tertiary sediment aquifers of northwestern Nigeria, using hydrochemical and isotopic techniques. In: Bocanegra E, Martínez D, Massone H (eds) Groundwater and human development, $\mathrm{pp}$ 907-915

7. Adelana SMA, Olasehinde PI, Vrbka P (2008) Isotope and geochemical characterization of surface and subsurface waters in the Semi-arid Sokoto basin, Nigeria. Afr J Sci Technol Sci Eng Ser 4(2):80-89

8. Adelana SMA, Vrbka P, Goni I, Edet A (2008) An overview of the geology and hydrogeology of Nigeria. In: Applied groundwater studies in Africa, Chapter 11, vol 15(11), pp 171-198. https:// doi.org/10.1201/9780203889497.ch11

9. Aeschbach-Hertig W, Gleeson T (2012) Regional strategies for the accelerating global problem of groundwater depletion. Nat Geosci 5(12):853-861. https://doi.org/10.1038/ngeo1617

10. Ahmed AA, Ali MH (2009) Hydrochemical evolution and variation of groundwater and its environmental impact at Sohag, Egypt. Arab J Geosci 4(3-4):339-352. https://doi.org/10.1007/ s12517-009-0055-z

11. Akinbiyi OA, Oladunjoye MA, Sanuade OA, Oyedeji O (2019) Geophysical characterization and hydraulic properties of unconsolidated floodplain aquifer system in Wamako area, Sokoto State, north-western Nigeria. Appl Water Sci 9(177):110. https://doi.org/10.1007/s13201-019-1065-y

12. Alagbe SA (2006) Preliminary evaluation of hydrochemistry of the Kalambaina Formation, Sokoto Basin, Nigeria. Environ Geol 51(1):39-45. https://doi.org/10.1007/s00254-006-0302-5

13. Anderson HR, Ogilbee W (1973) Aquifers in the Sokoto basin, Northwestern Nigeria, with a description of the general hydrogeology of the region: contributions to the hydrology of Africa and the Mediterranean Region. Geological Survey Water-Supply Paper 1757-L, pp 1-88

14. Ayadi R, Trabelsi R, Zouari K, Saibi H, Itoi R, Khanfir H (2017) Hydrogeological and hydrochemical investigation of groundwater using environmental isotopes $(18 \mathrm{O}, 2 \mathrm{H}, 3 \mathrm{H}, 14 \mathrm{C})$ and chemical tracers: a case study of the intermediate aquifer, Sfax, southeastern Tunisia. Hydrogeol J 26(4):983-1007. https://doi. org/10.1007/s10040-017-1702-1

15. Ayers RS, Westcot DW (1976) Water quality for agriculture. Food and Agriculture Organization of the United Nations Viale delle Terme di Caracalla 00153 Rome, Italy, Irrigation and Drainage Paper, pp 1-29

16. Azhar SC, Aris AZ, Yusoff MK, Ramli MF, Juahir H (2015) Classification of river water quality using multivariate analysis. Procedia Environ Sci 30:79-84. https://doi.org/10.1016/j.proen v.2015.10.014

17. Bahar MM, Reza MS (2010) Hydrochemical characteristics and quality assessment of shallow groundwater in a coastal area 
of Southwest Bangladesh. Environ Earth Sci 61(5):1065-1073. https://doi.org/10.1007/s12665-009-0427-4

18. Baker LA, Brezonik PL (1986) Sources and Sinks of ions in a soft water, acidic lake in Florida. Water Resour Res 22(5):715-722

19. Belkhiri L, Boudoukha A, Mouni L, Baouz T (2010) Application of multivariate statistical methods and inverse geochemical modeling for characterization of groundwater-a case study: Ain Azel plain (Algeria). Geoderma 159(3-4):390-398. https:// doi.org/10.1016/j.geoderma.2010.08.016

20. Belkhiri L, Mouni L, Tiri A (2012) Water-rock interaction and geochemistry of groundwater from the Ain Azel aquifer, Algeria. Environ Geochem Health 34(1):1-13. https://doi. org/10.1007/s10653-011-9376-4

21. Belkhiri L, Narany TS (2015) Using multivariate statistical analysis, geostatistical techniques and structural equation modeling to identify spatial variability of groundwater quality. Water Resour Manag 29:2073-2089. https://doi.org/10.1007/s1126 9-015-0929-7

22. Besser H, Mokadem N, Redhaounia B, Hadji R, Hamad A, Hamed $Y$ (2018) Groundwater mixing and geochemical assessment of low-enthalpy resources in the geothermal field of southwestern Tunisia. Euro Mediterr J Environ Integr 3(16):1-15. https:// doi.org/10.1007/s41207-018-0055-z

23. Besser H, Mokadem N, Redhouania B, Rhimi N, Khlifi F, Ayadi Y, Omar Z, Bouajila A, Hamed Y (2017) GIS-based evaluation of groundwater quality and estimation of soil salinization and land degradation risks in an arid Mediterranean site (SW Tunisia). Arab J Geosci 10(350):1-20. https://doi.org/10.1007/s1251 7-017-3148-0

24. Beyene G, Aberra D, Fufa F (2019) Evaluation of the suitability of groundwater for drinking and irrigation purposes in Jimma Zone of Oromia, Ethiopia. Groundw Sustain Dev 9(100216):1-8. https://doi.org/10.1016/j.gsd.2019.100216

25. Braune $E, X u Y(2010)$ The role of ground water in sub-Saharan Africa. Ground Water 48(2):229-238. https://doi.org/10.111 1/j.1745-6584.2009.00557.x

26. Chapman PJ, Reynolds B, Wheater HS (1997) Sources and controls of calcium and magnesium in storm runoff: the role of groundwater and ion exchange reactions along water flowpaths. Hydrol Earth Syst Sci 1(3):671-685

27. Chidambaram $S$, Anandhan $P$, Prasanna MV, Ramanathan AL, Srinivasamoorthy K, Senthil Kumar G (2012) Hydrogeochemical modelling for groundwater in Neyveli aquifer, Tamil Nadu, India, using PHREEQC: a case study. Nat Resour Res 21(3):311324. https://doi.org/10.1007/s11053-012-9180-6

28. Chiodini G, Frondini F, Cardellini C, Parello F, Peruzzi L (2000) Rate of diffuse carbon dioxide Earth degassing estimated from carbon balance of regional aquifers: the case of central Apennine, Italy. J Geophys Res Solid Earth 105(B4):8423-8434. https ://doi.org/10.1029/1999jb900355

29. Chitsazan M, Aghazadeh N, Mirzaee Y, Golestan Y (2017) Hydrochemical characteristics and the impact of anthropogenic activity on groundwater quality in suburban area of Urmia city, Iran. Environ Dev Sustain 21(1):331-351. https:// doi.org/10.1007/s10668-017-0039-1

30. Comte J-C, Cassidy R, Obando J, Robins N, Ibrahim K, Melchioly S, Mjemah I, Shauri H, Bourhane A, Mohamed I, Noe C, Mwega B, Makokha M, Join J-L, Banton O, Davies J (2016) Challenges in groundwater resource management in coastal aquifers of East Africa: investigations and lessons learnt in the Comoros Islands, Kenya and Tanzania. J Hydrol Reg Stud 5:179-199. https://doi. org/10.1016/j.ejrh.2015.12.065

31. Coomar P, Mukherjee A, Bhattacharya P, Bundschuh J, Verma S, Fryar AE, Ramos Ramos OE, Munoz MO, Gupta $S$, Mahanta C, Quino I, Thunvik R (2019) Contrasting controls on hydrogeochemistry of arsenic-enriched groundwater in the homologous tectonic settings of Andean and Himalayan basin aquifers, Latin America and South Asia. Sci Total Environ 689:1370-1387. https://doi.org/10.1016/j.scito tenv.2019.05.444

32. Danhalilu RL, Mustapha SM, Aliyu IK (2018) Groundwater quality in basement formation of Musawa Lga of Katsina State, North-Western Nigeria. Int J Adv Acad Res 4(4):95-105

33. Davis SN, Dewest RJ (1966) Hydrogeology. Wiley, New York, $p$ 463

34. De Caro M, Crosta GB, Frattini P (2017) Hydrogeochemical characterization and natural background levels in urbanized areas: Milan Metropolitan area (Northern Italy). J Hydrol 547:455-473. https://doi.org/10.1016/j.jhydrol.2017.02.025

35. de Graaf IEM, van Beek RLPH, Gleeson T, Moosdorf N, Schmitz O, Sutanudjaja EH, Bierkens MFP (2017) A global-scale two-layer transient groundwater model: development and application to groundwater depletion. Adv Water Resour 102:53-67. https:// doi.org/10.1016/j.advwatres.2017.01.011

36. Dugan HA, Summers JC, Skaff NK, Krivak-Tetley FE, Doubek JP, Burke SM, Bartlett SL, Arvola L, Jarjanazi H, Korponai J, Kleeberg A, Monet G, Monteith D, Moore K, Rogora M, Hanson PC, Weathers KC (2017) Long-term chloride concentrations in North American and European freshwater lakes. Sci Data 4:170101. https://doi.org/10.1038/sdata.2017.101

37. Egbueri JC (2020) Groundwater quality assessment using pollution index of groundwater (PIG), ecological risk index (ERI) and hierarchical cluster analysis (HCA): a case study. Groundw Sustain Dev 10(100292):1-8. https://doi.org/10.1016/j. gsd.2019.100292

38. Emeribe CN, Ogbomida ET, Enoma-Calus JO (2019) Climatic variability and estimation of supplementary irrigation water needs of selected food crops in the Sokoto-Rima River Basin, Nigeria. Niger. J. Environ. Sci. Technol. 3(1):86-104. https://doi. org/10.36263/nijest.2019.01.0111

39. Eniolorunda NB, Mashi SA, Nsofor GN (2016) Toward achieving a sustainable management: characterization of land use/land cover in Sokoto Rima floodplain, Nigeria. Environ Dev Sustain 19(5):1855-1878. https://doi.org/10.1007/s10668-016-9831-6

40. EPA (2001) Parameters of water quality: Interpretation and Standards. An Ghniomhaireacht um Chaomhnu Comhshaoil. Ireland. pp 1-132

41. Ferrandiz J, Abellan JJ, Gomez-Rubio V, Lopez-Quilez A, Sanmartin P, Abellan C, Martinez-Beneito MA, Melchor I, Vanaclocha H, Zurriaga O, Ballester F, Gil JM, Perez-Hoyos S, Ocana $R$ (2004) Spatial analysis of the relationship between mortality from cardiovascular and cerebrovascular disease and drinking water hardness. Environ Health Perspect 112(9):1037-1044. https://doi.org/10.1289/ehp.6737

42. Ganiyu SA, Badmus BS, Oladunjoye MA, Aizebeokhai AP, Ozebo VC, Idowu OA, Olurin OT (2016) Assessment of groundwater contamination around active dumpsite in Ibadan southwestern Nigeria using integrated electrical resistivity and hydrochemical methods. Environ Earth Sci. https://doi.org/10.1007/ s12665-016-5463-2

43. Geyh MA, Wirth K (1980) 14 C ages of confined groundwater from the Gwandu aquifer, Sokoto Basin, northern Nigeria. J Hydrol 48(3-4):281-288. https://doi.org/10.1016/00221694(80)90120-1

44. Ghandour MFM, Khalil JB, Atta SA (1985) Distribution of carbonates, bicarbonates, and ph values in ground water of the Nile Delta Region, Egypt. Ground Water 23(1):35-41

45. Gibbs RJ (1970) Mechanisms controlling world water chemistry. Science 170(3962):1088-1090 
46. Goni IB, Aji MM, Ibrahim M, Maduabuchi C, Kachallah M, Imam MK, Sulum M (2013) Geochemical studies of phreatic aquifer water in the Nigerian Sector of lullummeden Basin, NW Nigeria. J Min Geol 49(1):1-11

47. Gowing J, Parkin G, Forsythe N, Walker D, Haile AT, Alamirew D (2016) Shallow groundwater in sub-Saharan Africa: neglected opportunity for sustainable intensification of small-scale agriculture? Hydrol Earth Syst Sci Discuss. https://doi.org/10.5194/ hess-2015-549

48. Graham WBR, Pishiria IW, Ojo OI (2006) Monitoring of groundwater quality for small-scale irrigation: case studies in the southwest Sokoto-Rima Basin, Nigeria. Agric Eng Int CIGR E J 3:1-9

49. Hamad A, Baali F, Hadji R, Zerrouki H, Besser H, Mokadem N, Legrioui R, Hamed Y (2018) Hydrogeochemical characterization of water mineralization in Tebessa Kasserine karst system (Tuniso-Algerian Transboundry basin). Euro-Mediterr J Environ Integr 3(7):1-15. https://doi.org/10.1007/s41207-017-0045-6

50. Hamzah Z, Aris AZ, Ramli MF, Juahir H, Sheikhy Narany T (2017) Groundwater quality assessment using integrated geochemical methods, multivariate statistical analysis, and geostatistical technique in shallow coastal aquifer of Terengganu, Malaysia. Arab J Geosci 10(49):1-17. https://doi.org/10.1007/s1251 7-016-2828-5

51. Hokkanen S, Repo E, Westholm LJ, Lou S, Sainio T, Sillanpää M (2014) Adsorption of $\mathrm{Ni}^{2+}, \mathrm{Cd}^{2+}, \mathrm{PO}_{4}{ }^{3-}$ and $\mathrm{NO}_{3}{ }^{-}$from aqueous solutions by nanostructured microfibrillated cellulose modified with carbonated hydroxyapatite. Chem Eng J 252:64-74. https ://doi.org/10.1016/j.cej.2014.04.101

52. Hudak PF (2001) Water hardness and sodium trends in Texas aquifers. Environ Monit Assess 68:177-185

53. Isewede CO, Ozeto H, Azama AA, Jimoh O (2020) The level of iron in groundwater. Int J Earth Sci 2(1):1-14

54. Jankowski J, Beck P (2000) Aquifer heterogeneity: hydrogeological and hydrochemical properties of the Botany Sands aquifer and their impact on contaminant transport. Aust J Earth Sci 47:45-64

55. Jiang L, He P, Chen J, Liu Y, Liu D, Qin G, Tan N (2016) Magnesium levels in drinking water and coronary heart disease mortality risk: a meta-analysis. Nutrients 8(5):1-7. https://doi. org/10.3390/nu8010005

56. Jones B (1948) The sedimentary rocks of Sokoto Province. Niger Geol Surv Bull 18:1-75

57. Khazaei E, Milne-Home W (2017) Applicability of geochemical techniques and artificial sweeteners in discriminating the anthropogenic sources of chloride in shallow groundwater north of Toronto, Canada. Environ Monit Assess 189(189):1-13. https://doi.org/10.1007/s10661-017-5927-1

58. Kogbe CA (1975) Petrographic study of Maestriehtian and P-Pns of North-Western Nigeria (lullemmeden Basin). Geol Rundsch 64:216-229

59. Kogbe CA (1981) Cretaceous and tertiary of the iullemmeden Basin in Nigeria (West Africa). Cretac Res 2:129-186

60. Konikow LF, Kendy E (2005) Groundwater depletion: a global problem. Hydrogeol J 13(1):317-320. https://doi.org/10.1007/ s10040-004-0411-8

61. Lahav O, Birnhack L (2007) Quality criteria for desalinated water following post-treatment. Desalination 207(1-3):286-303. https://doi.org/10.1016/j.desal.2006.05.022

62. Li S, Park M-K, Jo CO, Park S (2016) Emission estimates of methyl chloride from industrial sources in China based on high frequency atmospheric observations. J Atmos Chem 74(2):227243. https://doi.org/10.1007/s10874-016-9354-4

63. Li X, Masuda H, Koba K, Zeng H (2006) Nitrogen isotope study on nitrate-contaminated groundwater in the Sichuan Basin,
China. Water Air Soil Pollut 178(1-4):145-156. https://doi. org/10.1007/s11270-006-9186-y

64. Li X, Wu H, Qian H, Gao Y (2018) Groundwater chemistry regulated by hydrochemical processes and geological structures: a case study in Tongchuan, China. Water 10(3):338. https://doi. org/10.3390/w10030338

65. Lin CY, Abdullah MH, Praveena SM, Yahaya AHB, Musta B (2012) Delineation of temporal variability and governing factors influencing the spatial variability of shallow groundwater chemistry in a tropical sedimentary island. J Hydrol 432-433:26-42. https ://doi.org/10.1016/j.jhydrol.2012.02.015

66. Liu C-W, Lin K-H, Kuo Y-M (2003) Application of factor analysis in the assessment of groundwater quality in a blackfoot disease area in Taiwan. Sci Total Environ 313(1-3):77-89. https://doi. org/10.1016/s0048-9697(02)00683-6

67. Liu J, Han G (2020) Effects of chemical weathering and $\mathrm{CO}_{2}$ outgassing on $\delta 13 \mathrm{CDIC}$ signals in a karst watershed. J Hydrol 589:125192. https://doi.org/10.1016/j.jhydrol.2020.125192

68. Liu XY, Xiao HW, Xiao HY, Song W, Sun XC, Zheng XD, Liu CQ, Koba K (2017) Stable isotope analyses of precipitation nitrogen sources in Guiyang, southwestern China. Environ Pollut 230:486-494. https://doi.org/10.1016/j.envpol.2017.06.010

69. Lo IM, Lam CS, Lai KC (2006) Hardness and carbonate effects on the reactivity of zero-valent iron for $\mathrm{Cr}(\mathrm{VI})$ removal. Water Res 40(3):595-605. https://doi.org/10.1016/j.watres.2005.11.033

70. Machiwal D, Cloutier V, Güler C, Kazakis N (2018) A review of GIS-integrated statistical techniques for groundwater quality evaluation and protection. Environ Earth Sci 77(681):1-30. https://doi.org/10.1007/s12665-018-7872-x

71. Machiwal D, Islam A, Kamble T (2019) Trends and probabilistic stability index for evaluating groundwater quality: the case of quaternary alluvial and quartzite aquifer system of India. J Environ Manag 237:457-475. https://doi.org/10.1016/j.jenvm an.2019.02.071

72. Magaritz M, Kafri U (1979) Concentration of magnesium in carbonate nodules of soils: an indication of fresh groundwater contamination by intruding seawater. Chem Geol 27:143-155

73. Marandi A, Shand P (2018) Groundwater chemistry and the Gibbs diagram. Appl Geochem 97:209-212. https://doi. org/10.1016/j.apgeochem.2018.07.009

74. Marghade D, Malpe DB, Zade AB (2011) Geochemical characterization of groundwater from northeastern part of Nagpur urban, Central India. Environ Earth Sci 62:1419-1430. https:// doi.org/10.1007/s12665-010-0627-y

75. Meybeck M (1987) Global chemical weathering of surficial rocks estimated from river dissolved loads. Am J Sci 287:401-428

76. Milewski A, Lezzaik K, Rotz R (2020) Sensitivity analysis of the groundwater risk index in the Middle East and North Africa Region. Environ Process 7(1):53-71. https://doi.org/10.1007/ s40710-019-00421-7

77. Moody RTJ (1997) In: Selley RC (eds) Chapter 5: African basins. Sedimentary basins of the world, 3 (series editor: K.J. Hsu), pp 89-103

78. Moody RTJ, Sutcliffe PJC (1991) The Cretaceous deposits of the lullemmeden Basin of Niger, central West Africa. Cretac Res 12:137-157

79. Mora A, Rosales-Lagarde L, Hernández-Antonio A, Mahlknecht J (2017) Hydrogeochemistry of groundwater supplied to the city of Monterrey, Mexico. Procedia Earth Planet Sci 17:356-359. https://doi.org/10.1016/j.proeps.2016.12.090

80. Mukate SV, Panaskar DB, Wagh VM, Baker SJ (2020) Understanding the influence of industrial and agricultural land uses on groundwater quality in semiarid region of Solapur, India. Environ Dev Sustain 22:3207-3238. https://doi.org/10.1007/s1066 8-019-00342-3 
81. Nagy J, Sipka S, SipkaJr S, Kocsis J, Horvath Z (2019) The hardness of drinking water negatively while socio-economic deprivation positively correlate with the age-adjusted mortality rates due to cardiovascular diseases in Hungarian Wine Regions. Int J Environ Res Public Health 16(3437):1-8. https:// doi.org/10.3390/ijerph16183437

82. Obaje NG (2009) Chapter 6: The Sokoto Basin (Nigerian sector of the lullemmeden Basin). Geology and Mineral Resources of Nigeria, Lecture notes in earth sciences, vol 120. Springer, Berlin, pp 77-89. https://doi.org/10.1007/978-3-540-92685-6

83. Offodile ME (2002) Groundwater study and development in Nigeria, 2nd edn. Mecon Geological and Engineering, Ltd Ehinder, Jos, p 453

84. Omonona OV, Okogbue CO (2016) Geochemistry of rare earth elements in groundwater from different aquifers in the Gboko area, central Benue Trough, Nigeria. Environ Earth Sci. https:// doi.org/10.1007/s12665-016-6329-3

85. Parker DH, Fargher MN, Carter JD, Turner DC (1964) Geological map of Nigeria. Nigeria Geological Survey series, 1: 250,000, sheet nos. 1, 2, 3, 6, 7 and 8 .

86. Parks S, Byrnes J, Abdelsalam MG, Laó Dávila DA, Atekwana EA, Atya MA (2017) Assessing groundwater accessibility in the Kharga Basin, Egypt: a remote sensing approach. J Afr Earth Sci 136:272-281. https://doi.org/10.1016/j.jafrearsci.2016.11.002

87. Piper AM (1944) A graphical procedure in the geochemical interpretation of water analysis. Am Geol Union 25:914-928

88. Rahman S, Mridha MK, Lee P, Ahme F (2018) Can taste rating of groundwater samples for the presence of iron be a novel approach to groundwater iron assessment? World Nutr 9(1):22-30

89. Rapant S, Cveckova V, Fajcikova K, Sedlakova D, Stehlikova B (2017) Impact of calcium and magnesium in groundwater and drinking water on the health of inhabitants of the Slovak Republic. Int J Environ Res Public Health 14(278):1-21. https:// doi.org/10.3390/ijerph14030278

90. Ravikumar P, Somashekar RK, Angami M (2011) Hydrochemistry and evaluation of groundwater suitability for irrigation and drinking purposes in the Markandeya River basin, Belgaum District, Karnataka State, India. Environ Monit Assess 173(14):459-487. https://doi.org/10.1007/s10661-010-1399-2

91. Saccani G, Hakanen J, Sindhya K, Ojalehto V, Hartikainen M, Antonelli M, Miettinen K (2019) Potential of interactive multiobjective optimization in supporting the design of a groundwater biodenitrification process. J Environ Manag 254:109770. https://doi.org/10.1016/j.jenvman.2019.109770

92. Saha P, Paul B (2019) Groundwater quality assessment in an industrial hotspot through interdisciplinary techniques. Environ Monit Assess 191(2):326. https://doi.org/10.1007/s1066 1-019-7418-z

93. Sawyer CN, McCarty PL (1967) Chemistry for sanitary engineers. Chemistry for sanitary engineers. McGraw-Hill, New York, pp $1-146$

94. Schoeller H (1965) Qualitative evaluation of groundwater resources. In: Methods and techniques of groundwater investigations and developments. UNESCO

95. Selvakumar S, Ramkumar K, Chandrasekar N, Magesh NS, Kaliraj S (2017) Groundwater quality and its suitability for drinking and irrigation use in southern Tiruchirappali district, Tamil Nadu, India. Appl Water Sci 7:411-420. https://doi.org/10.1007/ s13201-014-0256-9

96. Sethy SN, Syed TH, Kumar A, Sinha D (2016) Hydrogeological charaterization and quality assessment of groundwater in parts of southern Gangetic Plain. Environ Earth Sci 75(232):2-15. https://doi.org/10.1007/s12665-015-5049-4

97. Singh S, Raju NJ, Ramakrishna C (2015) Evaluation of groundwater quality and its suitability for domestic and irrigation use in parts of the Chandauli-Varanasi Region, Uttar Pradesh, India. J Water Resour Prot 07(07):572-587. https://doi.org/10.4236/ jwarp.2015.77046

98. Smith LED, Siciliano G (2015) A comprehensive review of constraints to improved management of fertilizers in China and mitigation of diffuse water pollution from agriculture. Agr Ecosyst Environ 209:15-25. https://doi.org/10.1016/j. agee.2015.02.016

99. Steven DB, Michael NJ (1978) Mass blooms of the Alga Cladophora in Bermuda. Mar Pollut Bull 9(2):34-37

100. Takdastan A, Mirzabeygi Radfard M, Yousefi M, Abbasnia A, Khodadadia R, Soleimani H, Mahvi AH, Naghan DJ (2018) Neuro-fuzzy inference system prediction of stability indices and sodium absorption ratio in Lordegan rural drinking water resources in west Iran. Data Brief 18:255-261. https://doi. org/10.1016/j.dib.2018.02.075

101. Teh T, Nik N, Nik AR, Shahadat M, Yong Y, Mohd OA (2016) Risk assessment of metal contamination in soil and groundwater in Asia: a review of recent trends as well as existing environmental laws and regulations. Pedosphere 26(4):431-450. https://doi. org/10.1016/s1002-0160(15)60055-8

102. Tiwari AK, Ghione R, De Maio M, Lavy M (2017) Evaluation of hydrogeochemical processes and groundwater quality for suitability of drinking and irrigation purposes: a case study in the Aosta Valley region, Italy. Arab J Geosci 10(264):1-18. https:// doi.org/10.1007/s12517-017-3031-z

103. Tolera MB, Choi H, Chang SW, Chung IM (2020) Groundwater quality evaluation for different uses in the lower Ketar Watershed, Ethiopia. Environ Geochem Health. https://doi. org/10.1007/s10653-019-00508-y

104. Usman AA, Gada MA, Bayawa AM, Dankani IM, Wali SU (2020) Examination of surface water along river-rima floodplain in Wamakko, Sokoto State, Nigeria. J Geol Res 2(3):43-51. https ://doi.org/10.30564/jgr.v2i3.2149

105. USSL (1954) United States salinity laboratory staff: diagnosis and improvements of saline and alkali soils, 60 . United States Department of Agriculture Handbook, Washington, pp 1-160

106. Wali SU, Dankani IM, Abubakar SD, Gada MA, Umar KJ, Usman AA, Shera IM (2020) Re-examination of hydrochemistry and groundwater potentials of Cross River and Imo-Kwa-lbo intersecting Tropical Basins of South-South Nigeria. J Geol Res 2(3):25-42. https://doi.org/10.30564/jgr.v2i3.2142

107. Wali SU, Umar K, Dankani IM, Abubar SD, Gada MA, Umar A, Usman AA (2018) Groundwater hydrochemical characterization in urban areas of Southwestern Sokoto Basin Nigeria. SF J Environ Earth Sci 1(1):1-17

108. Wali SU, Umar KJ, Abubakar SD, Ifabiyi IP, Dankani IM, Shera IB, Yauri SG (2019) Hydrochemical characterization of shallow and deep groundwater in Basement Complex areas of southern Kebbi State, Sokoto Basin, Nigeria. Appl Water Sci 9(169):1-36. https://doi.org/10.1007/s13201-019-1042-5

109. Wali SU, Umar KJ, Gada MA, Usman AA (2018) Evaluation of shallow groundwater in cretaceous and tertiary aquifers of Northern Kebbi State, Nigeria. SF J Environ Earth Sci 1(1):1-11

110. WHO (2018) Guidelines for drinking-water quality: fourth edition incorporating the first addendum. WHO library cataloguing-in-publication data. World Health Organization, Geneva, $p$ 631

111. Willhite CC, Bhat VS, Ball GL, McLellan CJ (2013) Emergency do not consume/do not use concentrations for potassium permanganate in drinking water. Hum Exp Toxicol 32(3):275-298. https://doi.org/10.1177/0960327112456316

112. Xue D, Botte J, De Baets B, Accoe F, Nestler A, Taylor P, Van Cleemput O, Berglund M, Boeckx P (2009) Present limitations and future prospects of stable isotope methods for nitrate 
source identification in surface- and groundwater. Water Res 43(5):1159-1170. https://doi.org/10.1016/j.watres.2008.12.048

113. Yang X, Wang T, Xia M, Gao X, Li Q, Zhang N, Gao Y, Lee S, Wang $X$, Xue L, Yang L, Wang W (2018) Abundance and origin of fine particulate chloride in continental China. Sci Total Environ 624:1041-1051. https://doi.org/10.1016/j.scito tenv.2017.12.205

114. Zhou Y, Li P, Xue L, Dong Z, Li D (2020) Solute geochemistry and groundwater quality for drinking and irrigation purposes: a case study in Xinle City, North China. Geochemistry. https:// doi.org/10.1016/j.chemer.2020.125609
115. Zielke-Olivier J, Vermeulen PD (2019) A geochemical weathering profile of a fine ash tailings dam and its impact on the underlying aquifers. J Environ Manag 242:162-170. https://doi. org/10.1016/j.jenvman.2019.04.034

Publisher's Note Springer Nature remains neutral with regard to jurisdictional claims in published maps and institutional affiliations. 